Open Peer Review on Qeios

\title{
The Implementation of Drug Checking Services for People Who Use Drugs: A Systematic Review
}

Nazlee Maghsoudi ${ }^{1}$, Justine Tanguay ${ }^{1}$, Kristy Scarfone ${ }^{1}$, Indhu Rammohan ${ }^{1}$, Carolyn Ziegler, Dan Werb², Ayden Scheim ${ }^{3}$

\author{
1 University of Toronto \\ 2 University of California, San Diego \\ 3 Drexel University
}

Funding: Support was provided by the Canadian Research Initiative in Substance Misuse, Canadian Institutes of Health Research (CIHR) Vanier Canada Graduate Scholarship, CIHR New Investigator Award, CIHR Fellowship, Ontario Ministry of Research, Innovation and Science Early Researcher Award, and St. Michael's Hospital Foundation.

Potential competing interests: No competing interests to declare.

\section{Abstract}

This preprint has been updated and formally published in Addiction (https://doi.org/10.1111/add.15734). A one-page fact sheet is also available.

Background and Aims: Drug checking services (DCS) provide people who use drugs (PWUD) with chemical analysis results of their drug samples, while simultaneously monitoring the unregulated drug market. We sought to identify and synthesize literature on the following domains: (a) influence of DCS on behaviour of PWUD; (b) monitoring of drug markets by DCS; and (c) outcomes related to models of DCS.

Methods: This review followed PRISMA guidelines and was pre-registered in PROSPERO (CRD42018105366). A systematic literature search was conducted in MEDLINE, Embase, Cochrane Central Register of Controlled Trials, Cochrane Database of Systematic Reviews, PsycINFO, Scopus, Web of Science, and Dissertations \& Theses Global. Eligible studies were peer-reviewed articles and conference abstracts, or grey literature, published in any language since 1990 and including original data on the domains. We assessed risk of bias for quantitative peer-reviewed articles reporting on behaviour or models of DCS using National Institutes of Health tools.

Results: We screened 2,463 titles and abstracts and 156 full-texts, with 90 studies meeting inclusion criteria. Most ( $n=65,72.2 \%)$ were from Europe and used cross-sectional designs $(n=79,87.7 \%)$. Monitoring of drug markets by DCS ( $n=63,70 \%$ ) was most commonly reported, followed by influence of DCS on behaviour $(n=31,34.4 \%)$ and outcomes related to models of DCS ( $n=17,18.9 \%)$. The most common outcome measures were detection of unexpected substances $(n=50,55.6 \%)$, expected substances ( $n=44,48.9 \%)$, new psychoactive substances $(n=40,44.4 \%)$, and drugs of concern $(n=32$, $36.5 \%)$ by DCS. 
Conclusions: Monitoring of drug markets by DCS is well established in Europe and increasingly in North America. There is an emerging evidence base demonstrating the capacity of DCS to influence behavioural intention, and a smaller subset of findings on its impact on the enacted behaviour of PWUD. Further research is needed on enacted behaviours and corresponding health outcomes including overdose, particularly among people who inject drugs or use opioids.

\section{INTRODUCTION}

A public health intervention operating for over fifty years, drug checking services (DCS) allow the public to submit drug samples from unregulated drug markets (i.e., illegal and legal drugs sold through criminal channels) for chemical analysis. DCS originated in California in the late 1960s and early 1970s during the rise of a psychedelic counterculture that championed the use of psychoactive substances to expand consciousness $(1,2)$. DCS were later adopted in European settings throughout the 1990s, beginning in the Netherlands, primarily in response to the popularity of dance events and associated use of MDMA and other drugs $(3,4)$. More recently, DCS have been implemented in Australasia, the Americas, and the United Kingdom (UK), often with an emphasis on preventing harms from new psychoactive substances (NPS), including synthetic opioids. A global review of DCS conducted in 2017 identified 31 services operating across 20 countries (5). Notably, the contamination of unregulated drug markets with fentanyl and the resulting opioid overdose crisis has motivated the recent expansion of DCS in Canada (6) and the United States (US) (7).

DCS provide people who use drugs (PWUD) with information on the chemical composition of their drug samples to facilitate more informed decision-making (8). While some analysis methods can be operated by PWUD, DCS typically offer tailored harm reduction advice with the provision of analysis results to PWUD (9). By aggregating data on the composition of drug samples, DCS provide insight into trends in the unregulated drug supply and inform policymaking and harm reduction activities at the population level (10). DCS can inform public health alerts (11) when drugs of concern are detected, thus offering potential benefits to the broader community of PWUD and service providers (12). DCS differ globally in terms of their legality and degree of government support, as well as where and how samples are collected and analyzed. Models include mobile services at events, fixed services where samples can be dropped off or mailed, and the distribution of analysis methods for personal use, all of which employ a variety of technologies with differing benefits and drawbacks $(8,13,14)$.

Given the growing availability of DCS and interest in their impacts, we conducted a systematic review to investigate, "What is known from the existing literature about the influence of DCS on PWUD?" Specifically, we synthesized evidence across three domains: (a)influence of DCS on behaviour of PWUD, (b) monitoring of drug markets by DCS, and (c) outcomes related to models of DCS. 


\section{METHODS}

The reporting of this systematic review was guided by the Preferred Reporting Items for Systematic Reviews and Meta-Analyses (PRISMA) (15). The protocol was registered in PROSPERO (CRD42018105366) (16).

\section{Search Strategy and Selection Criteria}

We developed, piloted, and refined the search strategy in consultation with a library sciences expert. The search strategy was peer-reviewed by a librarian outside the review team using the guideline for Peer Review of Electronic Search Strategies (PRESS) (17) and revised accordingly. We searched MEDLINE (OVID), Embase (OVID), Cochrane Central Register of Controlled Trials (OVID), Cochrane Database of Systematic Reviews (OVID), PsycINFO (OVID), Scopus, Web of Science (including Science Citation Index, Social Sciences Citation Index, Conference Proceedings Citation Index- Science, Conference Proceedings Citation Index- Social Science \& Humanities, Emerging Sources Citation Index), and Dissertations \& Theses Global (ProQuest) for peer-reviewed journal articles and conference abstracts published in any language from January 1, 1990 (the lower bound for when DCS proliferated) to July 26, 2018, with a full search update on October 16, 2019. We used medical subject headings (MeSH) and keywords, adapted for each database, related to DCS (including service names from a global review of DCS (5)), controlled drugs, and harm reduction services. To capture all domains, search terms specific to outcomes were not employed. The results were deduplicated in EndNote. The full search strategies for each database, exactly as run, appear in Appendix A. We also searched reference lists of included studies. Grey literature reporting on the influence of DCS on behaviour of PWUD was included and identified using Google, Google Scholar, and websites for DCS (5), as well as through contact with content experts. Search terms for Google and Google Scholar were "pill-testing" OR "pill testing" OR "drug-checking" OR "drug checking."

Eligibility criteria applied in the screening process were defined by Population, Intervention, and Evaluation (PIE). Studies were included if the population of interest was people of any age who engage in non-medical use of drugs and voluntarily access DCS. Studies that involved the implementation of DCS were included with no restrictions on analysis methods. Studies were excluded if the intervention involved analyzing human biological specimens, analysis results were not offered to clients, clients were not accessing DCS but independently employing analysis methods, or DCS were not implemented (e.g., feasibility study). Studies were included if they evaluated the (a) influence of DCS on behaviour of PWUD (broadly defined to include behavioural intentions and enacted behaviour), (b) monitoring of drug markets by DCS, and (c) outcomes related to models of DCS (including barriers and facilitators to use). Eligibility was restricted to studies reporting original quantitative or qualitative data. As such, commentaries, letters to the editor, editorials, reviews, and unpublished conference abstracts were excluded. Titles, abstracts, and full-texts were translated as needed. 


\section{Screening, Data Extraction, and Quality Assessment}

Screening and data extraction were conducted in DistillerSR (Evidence Partners, Ottawa, Ontario) using standardized, pilot-tested charting forms. The screening form contained questions based on the eligibility criteria and was used to assess the relevance of titles and abstracts, as well as full-texts. Two independent reviewers (NM and JT/IR) began by screening titles and abstracts. Any deemed relevant by at least one reviewer advanced to the next stage in which both reviewers (NM and JT/IR/KS) independently screened full-texts in duplicate for inclusion. Data extraction was then conducted in duplicate by both reviewers (NM and JT/KS). The data extraction form included intervention details (model of DCS, population), study characteristics (year, location, design), sample characteristics (type, size, age, sex), and findings for domains.

Risk of bias assessment was performed in duplicate for peer-reviewed articles reporting quantitative data on behaviour and/or models of DCS. Epidemiologic assessment of risk of bias was deemed inappropriate for drug market monitoring studies and other designs (e.g., qualitative research) (18). Tools from the National Institutes of Health were used to assess risk of bias for individual studies (see Appendix B). The Quality Assessment Tools for Observational Cohort and Cross-Sectional Studies (19) or for Before-After (Pre-Post) Studies with No Control Group (20) were used, as appropriate to study design. Studies were assigned one point for each criterion they satisfied and could receive up to 14 or 12 points for crosssectional and before-after studies respectively, with higher scores indicating less susceptibility to bias.

Conflicts at each stage were resolved by consensus between reviewers, with input from the last author as required. The last author triple-checked data extraction and quality assessment for $10 \%$ of studies.

\section{Data Analysis}

We used narrative synthesis without meta-analysis (21) for data analysis. Outcome measures were not pre-specified but inductively coded in an iterative process throughout data extraction to ensure all relevant outcomes of DCS were captured. Within each domain, study outcomes were compared with outcome measures identified in earlier studies. New outcome measures were added when findings were about a distinct construct. Specific data from studies were coded within the relevant outcome measure and summarized, such as by converting data points into ranges or condensing participant responses into a short sentence. The addition of new outcome measures decreased as data extraction proceeded and outcomes reached saturation. Analysis following data extraction led to the reorganization and addition of some outcome measures (e.g., separating intentions in response to actual or hypothetical analysis results from DCS, drugs of concern from other drugs detected). We derived and prioritized domains based on consultation with content experts and knowledge users through the Canadian Research Initiative in Substance Misuse (CRISM). We organized the narrative synthesis by outcome measures within each 
domain. For individual outcome measures, we summarized the range of findings and highlighted differences across settings and populations.

\section{RESULTS}

\section{Study Characteristics}

As shown in the PRISMA Flow Diagram (Figure 1), of 2,463 titles and abstracts and 156 full-texts assessed for relevance, 90 studies met the inclusion criteria (Appendix C). Primary reasons for exclusion at the fulltext screening stage were that the study did not report original research $(n=30)$, did not evaluate DCS as defined for this review $(n=19)$, or did not report on pre-specified domains $(n=17)$. Details of included studies are presented by domain in Supplementary Tables 1-3. Included studies were published between $1997-2019 ; 45.6 \%(n=41)$ were published in $2017-2019,25.6 \%(n=23)$ in $2014-2016$, and $28.9 \%(n=26)$ before 2014. Table 1 provides an overview of the most recent reported models of DCS, including analysis methods and populations.

Characteristics of included studies are described in Table 2, both overall and stratified by peer-reviewed articles $(n=54,60 \%)$, peer-reviewed conference abstracts $(n=19,21.1 \%)$, and grey literature $(n=17$, 18.9\%). Most studies ( $n=65,72.2 \%$ ) were from Europe (Austria, Belgium, France, Germany, Italy, Netherlands, Portugal, Slovenia, Spain, Switzerland, UK). Other countries represented included Australia, Canada, Colombia, Mexico, New Zealand, and the US. The majority of studies used cross-sectional $(n=49$, $54.4 \%)$ or repeated cross-sectional $(n=30,33.3 \%)$ designs. Monitoring of drug markets by DCS $(n=63$, $70 \%)$ was the most common domain, followed by influence of DCS on behaviour $(n=31,34.4 \%)$ and outcomes related to models of DCS $(n=17,18.9 \%)$.

\section{Outcome Measures}

Across 90 studies, we categorized 55 outcome measures (Figure 2). Outcome measures in each domain are presented in the following section. Findings related to the influence of DCS on behaviour, and common outcome measures related to drug market monitoring and models of DCS, are prioritized. Results of individual studies are in Supplementary Tables 1-3.

\section{Influence of DCS on Behaviour of PWUD}

The most common outcome measures related to the influence of DCS on behaviour were intent to use the analyzed substance ( $14.4 \%$ of studies, $n=13)$, influence of analysis results on drug use behaviour $(11.1 \%$, $n=10)$, and disposal of the analyzed substance $(8.9 \%, n=8)$. Enacted behaviours as observed or per selfreported historical recall were measured in 16 studies (17.8\%) (22-37). Intended behaviours in response to actual or hypothetical analysis results from DCS were assessed in 22 studies (24.4\%) (31-52).

Studies found DCS influenced intended behaviour and, although less researched, enacted behaviour. 
Among studies of PWUD in party settings (referred to as "partygoers" in studies), greater intention to not use the analyzed substance was consistently reported if analysis results were unexpected (33, 35, 40, 42, $43,45,48,52$ ) or "questionable"/"suspicious" (49-51). For example, a cross-sectional study from Australia ( $n=83$ ) in 2018 found partygoers were more likely to change their intention to use when analysis results were unexpected (Odds Ratio $[\mathrm{OR}]=2.63 ; 95 \%$ Confidence Interval $[\mathrm{Cl}]$ 0.85-8.16) (35), as did two crosssectional studies from Portugal $(n=310, n=100)$ in 2016 and $2014(40,43)$. Similarly, other intended behaviour changes - such as using less of a substance or seeking more information about it - were more common among partygoers when analysis results from DCS suggested substances were "questionable"/“suspicious" $(49,51)$.

The proportion of participants reporting analysis results from DCS influenced their drug use varied by population and setting. Among partygoers, 16\% of participants in the Netherlands in 1996 (29), 50\% in Austria in 1997-1999 (37), and 87\% in New Zealand ( $n=47)$ in 2018-2019 (33) reported analysis results impacted their drug use. A cross-sectional study from the US among people who inject drugs $(n=125)$ in 2017 found 43\% changed their behaviour, and this was more likely when fentanyl was detected (adjusted $\mathrm{OR}[\mathrm{aOR}]=5.08,95 \% \mathrm{Cl}=2.12-12.17)(22)$. Qualitative and longitudinal studies of young PWUD $(n=81)$ in the US in 2017 supported this finding and found fentanyl detection was associated with positive changes in overdose risk behaviours (i.e., using less, using with others, doing a test shot) $(31,34)$. Overall, and in alignment with findings on intended drug use behaviour in response to "questionable"/"suspicious" analysis results, self-reported behaviour was more likely to change when analysis results detected fentanyl. Beyond individual analysis results, a repeated cross-sectional study from Colombia $(n=1533)$ in 2013 and 2016 examined the influence of alerts from DCS and found a majority of partygoers reported an impact on their behaviour (36).

Only one study linked intended behaviours to observed health outcomes. A Canadian cross-sectional study of DCS at a supervised injection site $(n=1411)$ in 2016-2017 found people who inject drugs were more likely to report the intention to use a smaller quantity than usual when fentanyl was detected by DCS ( $\mathrm{OR}=9.36 ; 95 \% \mathrm{Cl}$ 4.25-20.65) (41). In turn, those intending to use a smaller quantity were found to be less likely to overdose $(\mathrm{OR}=0.41 ; 95 \% \mathrm{Cl} 0.18-0.89)$ and be administered naloxone $(\mathrm{OR}=0.38 ; 95 \% \mathrm{Cl} 0.15$ $0.96)$.

Disposal of the analyzed substance was observed $(24,26,27,32,35)$ or self-reported $(22,31,34)$ as an outcome of DCS in eight studies. Like other behaviours, disposal was more frequent when analysis results from DCS were unexpected $(24,27,32,52)$. Among partygoers in a cross-sectional study $(n=2078)$ in 2015 and case report ( $n=2786$ ) in 2014 from Canada, observed disposal ranged from 4\% (27) to 7\% (26). A longitudinal study from the US ( $n=81)$ in 2017 reported 10\% disposal among young PWUD when fentanyl was detected (34), while a cross-sectional study from the US $(n=125)$ in 2017 found $0 \%$ disposal among 
people who inject drugs (22). Qualitative research from Canada $(n=20)$ in 2017-2018 found intention to dispose varied by drug preference among structurally vulnerable (i.e., marginalized as a result of their positions in social hierarchies) PWUD, with those using opioids being less likely than those using stimulants to dispose if fentanyl was detected (38).

While three European studies and one Canadian study reported intent to sell the analyzed substance among partygoers, only one longitudinal study from the US $(n=81)$ in 2017 assessed this self-reported behaviour and found $10 \%$ of young PWUD sold the substance after fentanyl was detected (34).

With one exception (49) among the five studies assessing intent to share analysis results from DCS with others, the majority of partygoers reported an intention to do so. Two studies reporting on intent to share analysis results in a hypothetical situation found information sharing with friends and drug sellers was more common when results were "questionable"/"suspicious" $(49,51)$. Beyond sharing information, qualitative and longitudinal studies of young PWUD (n=81) in the US in 2017 found 58\% reported distributing fentanyl test strips received from DCS to others, particularly those perceived to have higher risk of using drugs containing fentanyl $(31,34)$.

While less directly relevant to the influence of DCS on behaviour, drug using patterns of partygoers who did and did not use DCS were compared in three European book-length cross-sectional studies undertaken in Belgium ( $n=486$ ) in 2006, Netherlands $(n=285)$ in 2002, and a multi-country study from Austria, Netherlands, and Germany ( $n=702)$ in 2002 (49-51). In all three studies, DCS clients reported higher frequencies of ecstasy use than non-clients. Furthermore, the multi-country study found the frequency of accessing DCS was negatively correlated with the frequency of ecstasy use and unsafe party behaviour (51). Patterns of accessing DCS were examined in 14 studies $(22,24,25,28,32,33,36,39-42$, $47,48,51$ ). With one exception (24) among reporting studies, the majority of partygoers accessed DCS for both themselves and other people $(40,47)$. Among partygoers, a cross-sectional study from Portugal ( $n=310)$ in 2016 reported $77 \%$ accessed DCS pre-use $(40)$ whereas a Colombian repeated crosssectional study ( $n=831$ ) in 2016 found $49 \%$ waited for their analysis results before using (36). Studies reporting on this outcome measure for people who inject drugs were mixed, with two cross-sectional studies from the US $(n=125, n=242)$ in 2017-2018 finding the majority accessed DCS pre-use $(22,25)$ and one Canadian cross-sectional study $(n=1411)$ in 2016-2017 reporting primarily post-use access $(41)$. In terms of receiving drug-related services (e.g., harm reduction, drug treatment) prior to accessing DCS, $70 \%$ or more of partygoers had not previously accessed drug-related services in four reporting studies ( 32 , $33,42,51)$. In contrast, only one cross-sectional study from the US among people who inject drugs $(n=125)$ in 2017 examined this pattern of accessing DCS and found a lesser majority of $54 \%$ were not an existing client of a syringe service program (22). 
Five studies reporting on behaviour referenced theories, models, or frameworks, including theories of reasoned action or planned behaviour $(40,44)$, the information-motivation-behavioral skills model $(31,34)$, and risk environment frameworks $(38,40)$.

\section{Drug Market Monitoring}

The most common outcome measures overall related to drug market monitoring and included: detection of unexpected substances ( $55.6 \%$ of studies, $n=50$ studies), expected substances $(48.9 \%, n=44)$, NPS $(44.4 \%, n=40)$, drugs of concern $(36.5 \%, n=32)$, and legal or no drugs $(28.9 \%, n=26)$ by DCS, and source of submitted substance $(18.9 \%, \mathrm{n}=17)$.

Outcome measures coded concordance as detection of the expected substance (i.e., expected drug only, expected drug with other unexpected drug) or of an unexpected substance (i.e., unexpected drug only) by DCS. These concordance measures were reported in $61.1 \%$ of studies $(n=55)$. Seven studies assessed concordance across multiple drug classes including psychedelics, stimulants, and depressants (40, 53-58); 11 studies focused exclusively on MDMA $(44,59-68)$. One study compared concordance between drugs purchased online and offline (69) and another compared concordance between controlled drugs and NPS (70).

NPS refer to "substances of abuse" that are not internationally controlled (e.g., synthetic cathinones, cannabinoids, opioids) (71). Four studies examining NPS were technical papers characterizing the chemistry of novel compounds (72-75). Only a single study outside of Europe reported on the detection of NPS (53). European studies reported increasing detection of NPS by DCS over time (55, 70, 76-84), with NPS presenting as expected $(55,70,78)$ and unexpected drugs $(55,79,80,82,84)$. A proliferation of different types of NPS, including cathinones and phenethylamines, was reported over time $(55,79,81$, 85).

Drugs of concern included fentanyl and analogues, as well as other drugs identified as causing health harms by included studies. Nine studies reported the detection of fentanyl or analogues by DCS in Canada $(24,41,53,54)$, Slovenia $(86)$, and Spain $(56,76,87)$ from $2016-2019$ and in the Netherlands in 2011 (58). Six of the studies reported that fentanyl or analogues were detected in samples expected to be other drugs $(24,41,54,58,76,87)$, and none reported fentanyl or analogues as expected. Other drugs of concern reported in 35 studies included atropine, DOx, levamisole, and P(M)MA; these were detected in Europe $(43,55-58,61,63,66-68,88,89)$, the US $(44,65)$, and Canada (53). Notably, some drugs of concern overlapped with NPS or legal drugs. Legal drugs (not including NPS) reported in ${ }^{3} 5$ studies were caffeine, ephedrine, levamisole, lidocaine, phenacetin, and procaine.

The source of substances submitted to DCS was an outcome in 17 studies, and was reported by individuals 
accessing DCS or based on where the sample was collected/mailed. Studies specified a location (e.g., region, country, city) $(44,56,60,65,70,72,76,81,90,91)$, online (e.g., webshops, cryptomarkets) (69, $75,81,87,91-93)$, or onsite/offsite music festival grounds where DCS were accessed $(26,32)$.

\section{Outcomes Related to Models of DCS}

Facilitators ( $12.2 \%$ of studies, $n=11)$ and barriers $(7.8 \%, n=7)$ to the use of DCS were the most common outcome measures related to models of DCS.Beyond the most common, this domain also included outcome measures on use, knowledge, perceptions, and preferences related to models of DCS.

Facilitators included motivations for use. Concerns about drug contents and negative health consequences from consumption were a primary facilitator to use of DCS. In the music festival setting, a cross-sectional study from the UK $(n=230)$ in 2016 found the most common motivator for accessing DCS was concern about the sample, including having already experienced negative effects (32). A time-series study conducted in the Netherlands $(n=22280)$ in 2004-2010 found people who use ecstasy more commonly reported health concerns as their rationale for accessing DCS after a drug market shortage of MDMA-like substances (94). Drug market changes and resulting concerns about drug contents and health consequences are highly relevant to other contexts as a facilitator for accessing DCS as well. Qualitative research in the US in 2017 reported concern about fentanyl in the unregulated drug market and associated risk of overdose as a facilitator for use of DCS among young PWUD $(n=81)(31)$, as well as people who use opioids ( $n=55$ ) (23). Related facilitators are distrust in drug markets $(31,86)$ and drug sellers (95). Unique facilitators among structurally vulnerable PWUD included allowing the provision of analysis results post-use and returning the sample after analysis (38). Select other facilitators to use of DCS were central location $(34,38)$ as well as accuracy and comprehensiveness of analysis results $(38,54)$.

Barriers to using DCS were often linked to described facilitators and included lack of concern over drug contents $(38,53)$, high trust in drug sellers $(95)$, inaccessible location $(38,86)$, and limitations of results $(23,38)$. Another barrier was legal risks due to drug criminalization $(31)$, which was linked to anonymity concerns (86). Legal risks and privacy concerns were also perceived barriers for people who sell drugs to access DCS (96).

\section{Study Quality}

Assessment of study quality was conducted for 13 articles meeting a priori criteria (Supplementary Tables 1 and 3). Among 11 cross-sectional studies, scores ranged from 3 to 7 of 14 possible points, with a mean of 4.8 (Standard Deviation=1.2). One time-series study received 5 points and one longitudinal study received 4 points, out of 12 possible points. All studies were of relatively poor quality and limitations related to cross-sectional study designs and an absence of clear, valid, reliable, and consistently implemented outcome measures. 


\section{DISCUSSION}

This systematic review identified 90 studies evaluating the impacts of DCS from 1990-2019. While scholarship is growing, knowledge gaps persist. Studies evaluating DCS were overwhelmingly geographically concentrated in Europe (72.2\%). Given most European DCS target PWUD in party settings, the available evidence on DCS is largely focused on this subpopulation. While these findings may not be generalizable (97) - particularly to different subpopulations of PWUD - recent expansion of DCS for overdose prevention in Canada and the US has led to an emergent evidence base on the impact of DCS on structurally vulnerable PWUD.

Monitoring of drug markets by DCS was the predominant domain reported in the literature. Strong evidence exists demonstrating that DCS provide a unique form of drug market monitoring by providing information on the level of concordance between expected (i.e., anticipated by individuals accessing DCS) and detected contents in drug samples. Available evidence also demonstrates the capacity of DCS to detect NPS and drugs of concern. Our primary domain - the influence of DCS on behaviour of PWUD - was measured in a third of studies, while behavioural intention in response to analysis results from DCS was assessed most often (24.4\%). Adjustments in behaviour were found across reporting studies, and were generally more common when results from DCS were unexpected or drugs of concern.

Given the proliferation of DCS since 1992 in non-English speaking countries, the comprehensiveness of this systematic review is a key strength achieved through inclusion of studies since 1990 in all languages, grey literature, and peer-reviewed conference abstracts. This work has limitations, however, typical of systematic reviews. Publication bias may limit the representativeness of the included literature, as studies not showing positive impacts of DCS could be underrepresented. In addition, due to resource limitations, we only reviewed grey literature on the primary domain and did not appraise the quality of grey literature or qualitative studies. Studies for which quality was assessed were not of high quality, which may increase the level of uncertainty with respect to outcomes reported. Key methodological limitations among studies reporting on behaviour of PWUD include cross-sectional designs and behavioural intention measures subject to the intention-behaviour gap (98). Challenges facing the generation of evidence on DCS include limited resources for research and evaluation as well as barriers to conducting research on PWUD and DCS due to drug criminalization (99).

\section{Conclusions}

This systematic review found that monitoring of drug markets by DCS is well established in Europe and increasingly in North America, and there is an emerging evidence base on the capacity of DCS to influence behavioural intention, and to a lesser extent, enacted behaviour, among PWUD. Further research on enacted behaviours, linking behaviours to health outcomes, and among people who inject drugs or use 
opioids would benefit the knowledge base, as would more rigorous and higher quality study designs. As DCS gain popularity, ongoing scientific evaluations across settings are critical to understanding the impact and limitations of this intervention.

\section{ACKNOWLEDGEMENTS}

Support was provided by CRISM, Canadian Institutes of Health Research (CIHR) Vanier Canada Graduate Scholarship (NM), CIHR New Investigator Award (DW), CIHR Fellowship (AS), Ontario Ministry of Research, Innovation and Science Early Researcher Award (DW), and St. Michael's Hospital Foundation.

We acknowledge the leadership and working group of CRISM Emerging Health Threat Implementation Science Program on Opioid Interventions and Services: Drug Checking.

We acknowledge the land where we worked is the traditional territory of many nations including the Mississaugas of the Credit, Anishnabeg, Chippewa, Haudenosaunee, and Wendat peoples, and home to many diverse First Nations, Inuit, and Métis peoples.

A preprint of this manuscript appears at [URL].

\section{REFERENCES}

1. Smith D. Street drug analysis and community based drug programs. Journal of Psychedelic Drugs. $1974 ; 6(2): 153-9$.

2. Renfroe C. MDMA on the street: Analysis Anonymous. Journal of Psychoactive Drugs. 1986;18(4):363-9.

3. Kriener H, Billeth R, Gollner C, Lachout S, Neubauer P, Schmid R. An inventory of on-site pill-testing interventions in the EU. Vienna, Austria: European Monitoring Centre for Drugs and Drug Addiction. 2001.

4. Brunt T. Drug checking as a harm reduction tool for recreational drug users: opportunities and challenges. European Monitoring Centre on Drugs and Drug Addiction; 2017.

5. Barratt M, Kowalski M, Maier L, Ritter A. Global review of drug checking services operating in 2017. Sydney, Australia: National Drug and Alcohol Research Centre, UNSW Sydney; 2018.

6. Maghsoudi N, McDonald K, Stefan C, Beriault D, Mason K, Barnaby L, et al. Evaluating networked drug checking services in Toronto, Ontario: study protocol and rationale. Harm Reduction Journal. 2020;17(1):110.

7. Fentanyl Overdose Reduction Checking Analysis Study (FORECAST). Johns Hopkins Bloomberg School of Public Health and Bloomberg American Health Initiative; 2018.

8. Kerr T, Tupper K. Drug checking as a harm reduction intervention: British Columbia Centre on Substance Use; 2017.

9. Leece P. Evidence Brief: Drug checking services as a harm reduction intervention. Public Health Ontario; 2017. 
10. Schroers A. Drug checking: monitoring the contents of new synthetic drugs. Journal of Drug Issues. 2002;32(2):635-46.

11. Factsheet on drug checking in Europe. Nightlife Empowerment \& Well-being Implementation Project and Trans European Drug Information; 2011.

12. Drug checking service: good practice standards. Nightlife Empowerment \& Well-being Implementation Project; 2012.

13. Guidelines for drug checking methodology. Nightlife Empowerment \& Well-being Implementation Project and Trans European Drug Information; 2012.

14. Harper L, Powell J, Pijl EM. An overview of forensic drug testing methods and their suitability for harm reduction point-of-care services. Harm Reduction Journal. 2017;14(1):52.

15. Page M, McKenzie J, Bossuyt P, Boutron I, Hoffmann T, Mulrow C, et al. The PRISMA 2020 statement: an updated guideline for reporting systematic reviews. BMJ. 2021;372:n71.

16. Maghsoudi N, Werb D, Scheim A, Tanguay J, Ziegler C. The implementation of drug checking services for people who use drugs: a systematic review. PROSPERO: International prospective register of systematic reviews. 2018. CRD42018105366. Available from: https://www.crd.york.ac.uk/prospero/display_record.php? RecordID $=105366$.

17. McGowan J, Sampson M, Salzwedel D, Cogo E, Foerster V, Lefebvre C. PRESS Peer Review of Electronic Search Strategies: 2015 Guideline Statement. Journal of Clinical Epidemiology. 2016;75:40-6.

18. Ma L-L, Wang Y-Y, Yang Z-H, Huang D, Weng H, Zeng X-T. Methodological quality (risk of bias) assessment tools for primary and secondary medical studies: what are they and which is better? Military Medical Research. 2020;7(1):7.

19. Quality Assessment Tool for Observational Cohort and Cross-sectional Studies. National Institutes of Health; 2014.

20. Quality Assessment Tool for Before-After (Pre-Post) Studies With No Control Group. National Institutes of Health; 2014.

21. Popay J, Roberts H, Sowden A, Petticrew M, Arai L, Rodgers M, et al. Guidance on the conduct of narrative synthesis in systematic reviews. A product from the ESRC methods programme Version. 2006;1:b92.

22. Peiper N, Clarke S, Vincent L, Ciccarone D, Kral A, Zibbell J. Fentanyl test strips as an opioid overdose prevention strategy: findings from a syringe services program in the Southeastern United States. International Journal of Drug Policy. 2019;63:122-8.

23. McKnight C, Des Jarlais D. Being "hooked up" during a sharp increase in the availability of illicitly manufactured fentanyl: adaptations of drug using practices among people who use drugs (PWUD) in New York City. International Journal of Drug Policy. 2018;60:82-8.

24. Mema S, Sage C, Xu Y, Tupper K, Ziemianowicz D, McCrae K, et al. Drug checking at an electronic dance music festival during the public health overdose emergency in British Columbia. Canadian Journal of Public Health. 2018;109:740-4. 
25. Fentanyl Test Strip Pilot: San Francisco August 2017 - January 2018. Harm Reduction Coalition; 2018. 26. Munn M, Lund A, Golby R, Turris S. Observed benefits to on-site medical services during an annual 5day electronic dance music event with harm reduction services. Prehospital and Disaster Medicine. 2016;31(2):228-34

27. Sage C. Harm Reduction and Drug Checking; A wrap-around service for festivals. Case Study: Shambhala Music Festival /ANKORS Drug Checking Harm Reduction Service data 2015. ANKORS (AIDS Network Outreach \& Support Society); 2016.

28. Huberty C, Favresse D. Evaluation des actions de réduction des risques dans le cadre des activités de testing menées par I'ASBL Modus Vivendi: Rapport de recherche. 2010.

29. van de Wijngaart G, Braam R, de Bruin D, Fris M, Maalsté N, Verbraeck H. Ecstasy use at large-scale dance events in the Netherlands. Journal of Drug Issues. 1999;29(3):679-701.

30. van de Wijngaart G, Braam R, de Bruin D, Fris M, Maalsté N, Verbraeck H. Ecstasy and the Dutch rave scene: A socio-epidemiological study on the nature and extent of, and the risks involved in using ecstasy and other party drugs at dance events. Addiction Research Centre. 1998.

31. Goldman J, Waye K, Periera K, Krieger M, Yedinak J, Marshall B. Perspectives on rapid fentanyl test strips as a harm reduction practice among young adults who use drugs: a qualitative study. Harm Reduction Journal. 2019;16(3):1-11.

32. Measham F. Drug safety testing, disposals and dealing in an English field: exploring the operational and behavioural outcomes of the UK's first onsite 'drug checking'service. International Journal of Drug Policy. 2019;67:102-7.

33. 2018/2019 Results. KnowYourStuffNZ; 2019.

34. Krieger M, Goedel W, Buxton J, Lysyshyn M, Bernstein E, Sherman S, et al. Use of rapid fentanyl test strips among young adults who use drugs. International Journal of Drug Policy. 2018;61:52-8.

35. Makkai T, Macleod M, Vumbaca G, Hill P, Caldicott D, Noffs M, et al. Report on Canberra GTM Harm Reduction Service. Harm Reduction Australia; 2018.

36. Morris V, Gordillo C. Informe de Resultados Encuesta de Satisfacción y Cambio de Comportamiento Échele Cabeza 2016. Échele Cabeza; 2016.

37. Kriener H, Schmid R. Check your pills. Check your life. ChEck iT! High quality on-site testing of illicit substances. Information, counselling and safer use measures at raves in Austria. 2005.

38. Bardwell G, Boyd J, Tupper K, Kerr T. "We don't got that kind of time, man. We're trying to get high!": Exploring potential use of drug checking technologies among structurally vulnerable people who use drugs. International Journal of Drug Policy. 2019;71:125-32.

39. Sherman S, Morales K, Park J, McKenzie M, Marshall B, Green T. Acceptability of implementing community-based drug checking services for people who use drugs in three United States cities: Baltimore, Boston and Providence. International Journal of Drug Policy. 2019;68:46-53.

40. Valente H, Martins D, Carvalho H, Pires C, Carvalho M, Pinto M, et al. Evaluation of a drug checking service at a large scale electronic music festival in Portugal. International Journal of Drug Policy. 
2019;73:88-95.

41. Karamouzian M, Dohoo C, Forsting S, McNeil R, Kerr T, Lysyshyn M. Evaluation of a fentanyl drug checking service for clients of a supervised injection facility, Vancouver, Canada. Harm Reduction Journal. 2018;15(46):1-8

42. 2017/2018 Results. KnowYourStuffNZ; 2018.

43. Martins D, Barratt M, Pires C, Carvalho H, Ventura M, Fornís I, et al. The detection and prevention of unintentional consumption of DOx and 25x-NBOMe at Portugal's Boom Festival. Human Psychopharmacology: Clinical and Experimental. 2017;32:E2608.

44. Saleemi S, Pennybaker S, Wooldridge M, Johnson M. Who is 'Molly'? MDMA adulterants by product name and the impact of harm-reduction services at raves. Journal of Psychopharmacology.

2017;31(8):1056-60.

45. 2016/2017 Results. KnowYourStuffNZ; 2017.

46. Drug safety testing at festivals and night clubs. Royal Society for Public Health; 2017.

47. Michelow W, Dowden C. "Start Small, Take it Easy": Results from the ANKORS Harm Reduction Survey at the 2013 Shambhala Music Festival. ANKORS (AIDS Network Outreach \& Support Society); 2015. 48. Italian Results. BAONPS (Be Aware On Night Pleasure Safety); 2014.

49. Houioux G, Favresse D, De Smet P, Piette D. Réduction des risques avec analyse de nouvelles drogues de synthèse: Evaluation. 2006.

50. Korf D, Benschop A, Brunt T. Pillen testen in Nederland: Een onderzoek naar versterking van de monitor uitgaansdrugs: Rozenberg Publishers; 2003.

51. Benschop A, Rabes M, Korf D. Pill Testing, Ecstasy \& Prevention: A Scientific Evaluation in Three European Cities: Rozenberg Publishers; 2002.

52. Koeter M. Laat je xtc testen!: Evaluatie van de testservice van de Brijder Stichting: Amsterdam Institute for Addiction Research; 1997.

53. McCrae K, Tobias S, Tupper K, Arredondo J, Henry B, Mema S, et al. Drug checking services at music festivals and events in a Canadian setting. Drug and Alcohol Dependence. 2019;205:107589.

54. Tupper K, McCrae K, Garber I, Lysyshyn M, Wood E. Initial results of a drug checking pilot program to detect fentanyl adulteration in a Canadian setting. Drug and Alcohol Dependence. 2018;190:242-5.

55. Brunt T, Nagy C, Bücheli A, Martins D, Ugarte M, Beduwe C, et al. Drug testing in Europe: monitoring results of the Trans European Drug Information (TEDI) project. Drug Testing and Analysis. 2017;9(2):18898.

56. Caudevilla F, Ventura M, Fornís I, Barratt M, Vidal C, Quintana P, et al. Results of an international drug testing service for cryptomarket users. International Journal of Drug Policy. 2016;35:38-41.

57. Martins D, Valente H, Pires C. CHECK!NG: the last frontier for Harm Reduction in party settings. Saúde e Sociedade. 2015;24(2):646-60.

58. Brunt T, Niesink R. The Drug Information and Monitoring System (DIMS) in the Netherlands: implementation, results, and international comparison. Drug Testing and Analysis. 2011;3(9):621-34. 
59. Vidal C, Ventura M, Fornís I, Gil C, Calzada N, Fitó A, et al. Crystals and tablets in the Spanish ecstasy market 2000-2014: are they the same or different in terms of purity and adulteration? Forensic Science International. 2016;263:164-8.

60. Sibbald K, Rushton W, King J, Charlton N. Adulterants in tablets sold as Ecstasy in the US from 20092013. Clinical Toxicology. 2014;52(7):704-5.

61. Brunt T, Koeter M, Niesink R, van den Brink W. Linking the pharmacological content of ecstasy tablets to the subjective experiences of drug users. Psychopharmacology. 2012;220(4):751-62.

62. Brunt T, Poortman A, Niesink R, van den Brink W. Instability of the ecstasy market and a new kid on the block: mephedrone. Journal of Psychopharmacology. 2011;25(11):1543-7.

63. Vogels N, Brunt T, Rigter S, van Dijk P, Vervaeke H, Niesink R. Content of ecstasy in the Netherlands: 1993-2008. Addiction. 2009;104:2057-66.

64. Giraudon I, Bello P-Y. Monitoring ecstasy content in France: results from the National Surveillance System 1999-2004. Substance Use \& Misuse. 2007;42(10):1567-78.

65. Tanner-Smith E. Pharmacological content of tablets sold as "ecstasy": results from an online testing service. Drug and Alcohol Dependence. 2006;83(3):247-54.

66. Delile J-M, Gachie J-P. Ecstasy et réduction des risques: La place du testing et des analyses de substances. Alcoologie et Addictologie. 2002;24(4):311-8.

67. Spruit I. Monitoring synthetic drug markets, trends, and public health. Substance Use \& Misuse. 2001;36(1-2):23-47.

68. Spruit I. Ecstasy use and policy responses in the Netherlands. Journal of Drug Issues. 1999;29(3):65377.

69. van der Gouwe D, Brunt T, van Laar M, van der Pol P. Purity, adulteration and price of drugs bought online versus off-line in the Netherlands. Addiction. 2017;112:640-8.

70. Palma A, Ventura M, Galindo L, Fonseca F, Grifell M, Quintana P, et al. Something new about something old: a 10-year follow-up on classical and new psychoactive tryptamines and results of analysis. Journal of Psychoactive Drugs. 2017;49(4):297-305.

71. The challenge of new psychoactive substances. United Nations Office on Drugs and Crime; 2013.

72. Fabregat-Safont D, Carbón X, Ventura M, Fornís I, Hernández F, Ibáñez M. Characterization of a recently detected halogenated aminorex derivative: para-fluoro-4-methylaminorex (4' F-4-MAR). Scientific Reports. 2019;9(1):1-8.

73. Fabregat-Safont D, Carbón X, Gil C, Ventura M, Sancho J, Hernández F, et al. Reporting the novel synthetic cathinone 5-PPDI through its analytical characterization by mass spectrometry and nuclear magnetic resonance. Forensic Toxicology. 2018;36(2):447-57.

74. Fabregat-Safont D, Carbón X, Ventura M, Fornís I, Guillamón E, Sancho J, et al. Updating the list of known opioids through identification and characterization of the new opioid derivative 3, 4-dichloro- $\mathrm{N}$-(2(diethylamino) cyclohexyl)-N-methylbenzamide (U-49900). Scientific Reports. 2017;7(1):1-14. 75. Fabregat-Safont D, Fornís I, Ventura M, Gil C, Calzada N, Sancho J, et al. Identification and 
characterization of a putative new psychoactive substance, 2-(2-(4-chlorophenyl) acetamido)-3methylbutanamide, in Spain. Drug Testing and Analysis. 2017;10.

76. Roldán M, Grifell M, Gonzalez I, Fuentes J, Frings M, Ventura M, et al. Analysing heroin samples as harm reduction intervention - prevalence of adulteration with fentanyl derivatives. European Neuropsychopharmacology. 2019;29:S474-S5.

77. Pérez S, de Dios M, Monteagudo E, Sanagustín D, Trabsa A, Grifell M, et al. New designer benzodiazepines use in Barcelona. European Psychiatry. 2017;41(S1):S874.

78. Linsen F, Koning R, van Laar M, Niesink R, Koeter M, Brunt T. 4-Fluoroamphetamine in the Netherlands: more than a one-night stand. Addiction. 2015;110(7):1138-43.

79. Hondebrink L, Nugteren-van Lonkhuyzen J, Van Der Gouwe D, Brunt T. Monitoring new psychoactive substances (NPS) in The Netherlands: data from the drug market and the Poisons Information Centre. Drug and Alcohol Dependence. 2015;147:109-15.

80. Vidal C, Fornís I, Ventura M. New psychoactive substances as adulterants of controlled drugs. A worrying phenomenon? Drug Testing and Analysis. 2014;6(7-8):819-24.

81. Caudevilla F, Ventura M, Indave B, Fornís I. Presence and composition of cathinone derivatives in drug samples taken from a drug test service in Spain (2010-2012). Human Psychopharmacology: Clinical and Experimental. 2013;28(4):341-4.

82. Blanckaert P, van Amsterdam J, Brunt T, van den Berg J, Van Durme F, Maudens K, et al. 4-Methylamphetamine: a health threat for recreational amphetamine users. Journal of Psychopharmacology. 2013;27(9):817-22.

83. Caudevilla F, Riba J, Ventura M, González D, Farré M, Barbanoj M, et al. 4-Bromo-2, 5dimethoxyphenethylamine (2C-B): presence in the recreational drug market in Spain, pattern of use and subjective effects. Journal of Psychopharmacology. 2012;26(7):1026-35.

84. Bossong M, Brunt T, Van Dijk J, Rigter S, Hoek J, Goldschmidt H, et al. mCPP: an undesired addition to the ecstasy market. Journal of Psychopharmacology. 2010;24(9):1395-401.

85. Grifell M, Ventura M, Carbón X, Quintana P, Galindo L, Palma A, et al. Patterns of use and toxicity of new para-halogenated substituted cathinones: 4-CMC (clephedrone), 4-CEC (4-chloroethcatinone) and 4BMC (brephedrone). Human Psychopharmacology: Clinical and Experimental. 2017;32:E2621.

86. Sande M, Šabić S. The importance of drug checking outside the context of nightlife in Slovenia. Harm Reduction Journal. 2018;15(2):1-8.

87. Quintana P, Ventura M, Grifell M, Palma A, Galindo L, Fornís I, et al. The hidden web and the fentanyl problem: detection of ocfentanil as an adulterant in heroin. International Journal of Drug Policy. 2017; 40:78-83.

88. Grifell M, Palma A, Ventura M, Fornís I, Dinamarca F, Torrens M, et al. A trip to the unknown: 2, 5Dimethoxy-4-chloroamphetamine (DOC) sold as LSD: study on samples delivered by users asking for substance analysis. European Neuropsychopharmacology. 2015;25:S620.

89. Brunt T, Rigter S, Hoek J, Vogels N, van Dijk P, Niesink R. An analysis of cocaine powder in the 
Netherlands: content and health hazards due to adulterants. Addiction. 2009;104(5):798-805.

90. Sanagustín D, de Dios M, Monteagudo E, Pérez S, Trabsa A, Galindo L, et al. U-47700: the new emerging opioid drug. European Psychiatry. 2017;41(S1):S395.

91. Galindo L, Grifell M, Quintana P, Palma A, Tirado J, Ventura M, et al. The synthetic cannabinoids: JWH, four years of analysis. European Psychiatry. 2016;33(S1):S115-S6.

92. Monteagudo E, de Dios M, Trabsa A, Grifell M, Galindo L, Quintana P, et al. Is methylone a new public health threat in Spain? European Psychiatry. 2017;41(S1):S871.

93. Brunt T, Atkinson A, Nefau T, Martinez M, Lahaie E, Malzcewski A, et al. Online test purchased new psychoactive substances in 5 different European countries: a snapshot study of chemical composition and price. International Journal of Drug Policy. 2017;44:105-14.

94. Brunt T, Niesink R, van den Brink W. Impact of a transient instability of the ecstasy market on health concerns and drug use patterns in The Netherlands. International Journal of Drug Policy. 2012;23(2):13440.

95. Bardwell G, Boyd J, Arredondo J, McNeil R, Kerr T. Trusting the Source: The Potential Role of Drug Dealers in Reducing Drug-related Harms via Drug Checking. Drug and alcohol dependence. 2019;198:1-6. 96. Palamar J, Acosta P, Sutherland R, Shedlin M, Barratt M. Adulterants and altruism: a qualitative investigation of "drug checkers" in North America. International Journal of Drug Policy. 2019;74:160-9. 97. Palamar J, Fitzgerald N, Keyes K, Cottler L. Drug checking at dance festivals: A review with recommendations to increase generalizability of findings. Experimental and Clinical Psychopharmacology. 2021:No Pagination Specified-No Pagination Specified.

98. Sheeran P, Webb T. The intention-behavior gap. Social and Personality Psychology Compass. 2016;10(9):503-18.

99. Vidal C, Ventura M, Measham F, Brunt T, Bücheli A, Paulos C, et al. The utility of drug checking services as monitoring tools and more: A response to Pirona et al. International Journal of Drug Policy. 2017;45:46-7.

FIGURES AND TABLES

Figure 1: PRISMA Flow Diagram 


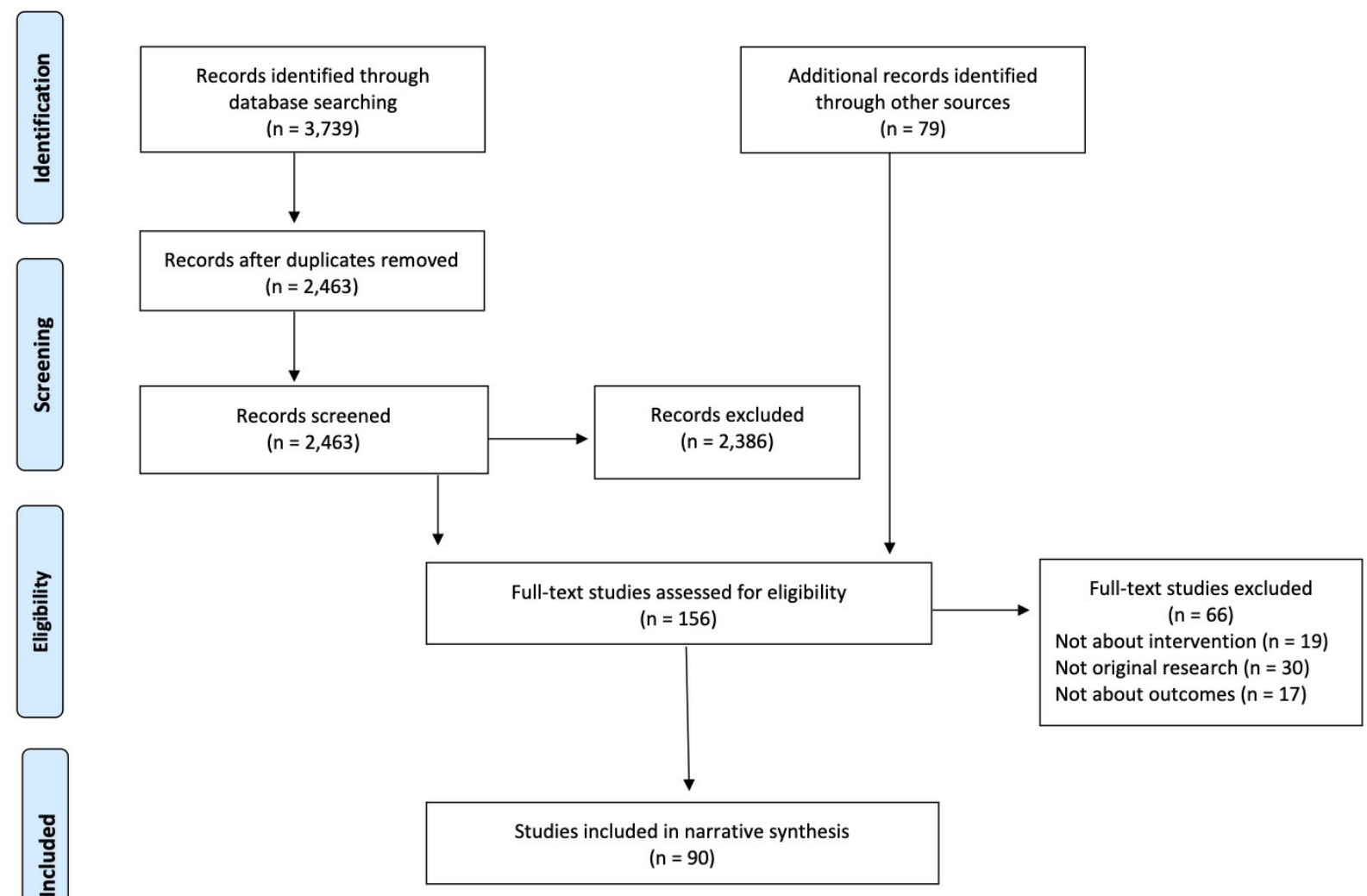

Figure 2: Outcome Measures Examined by Included Studies $(n=90)$ 
Figure 2A. Influence of DCS on Behaviour of PWUD ( $n=31$ studies)
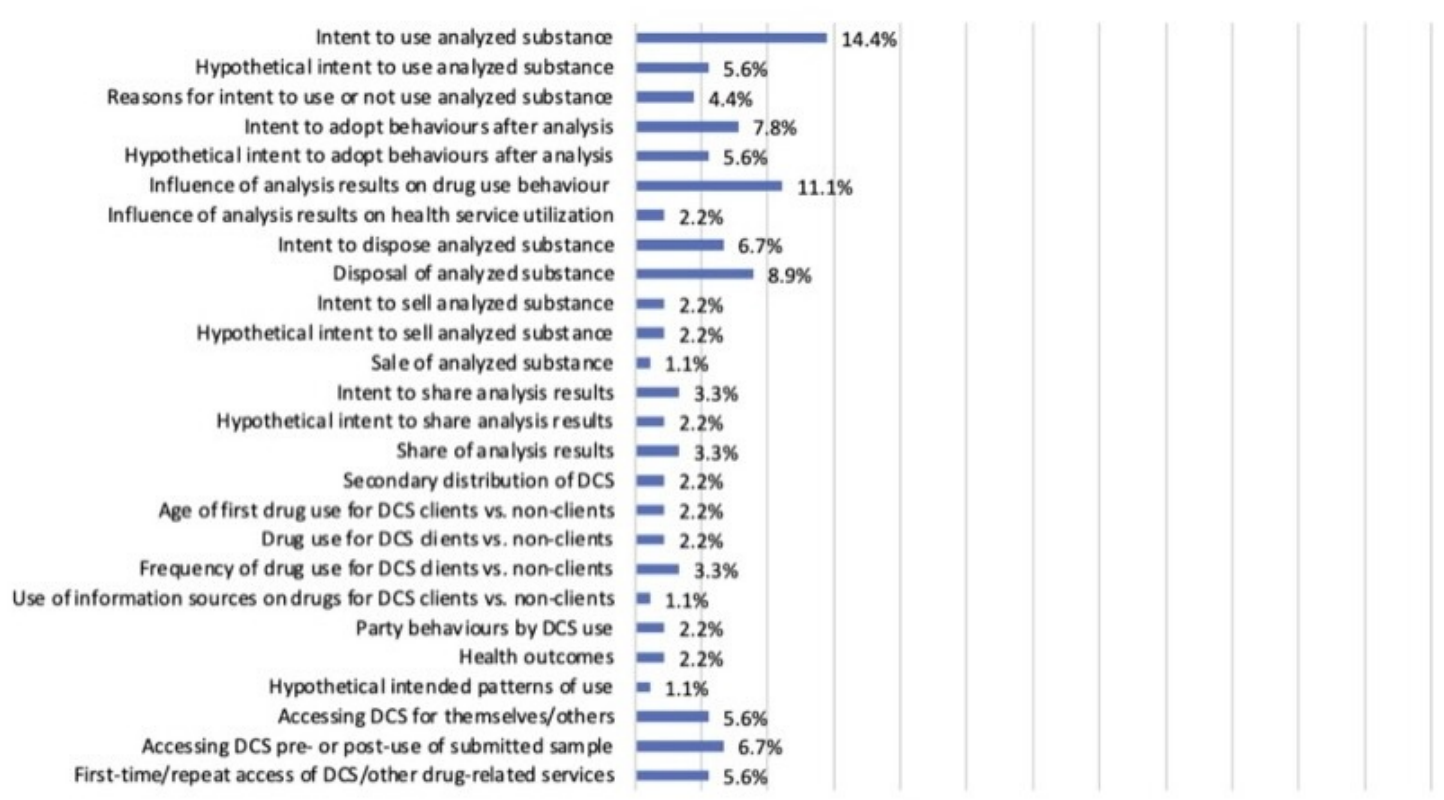

Figure 2B. Monitoring of Drug Markets by DCS ( $n=63$ studies)

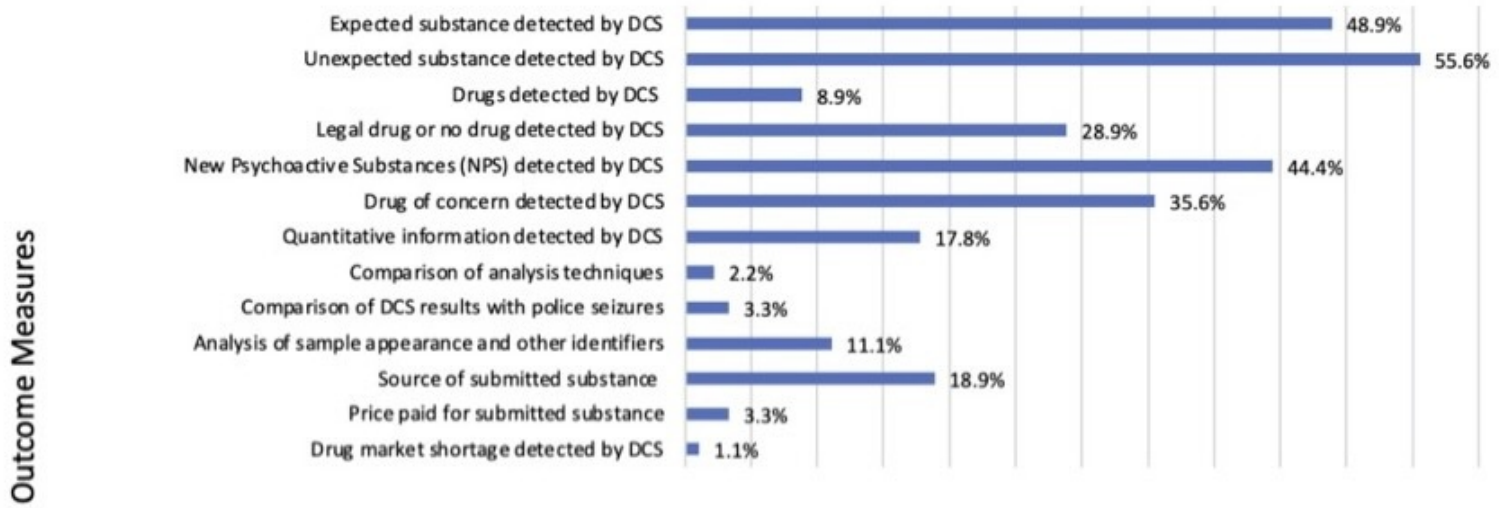

Figure 2C. Outcomes Related to Models of DCS ( $n=17$ studies)

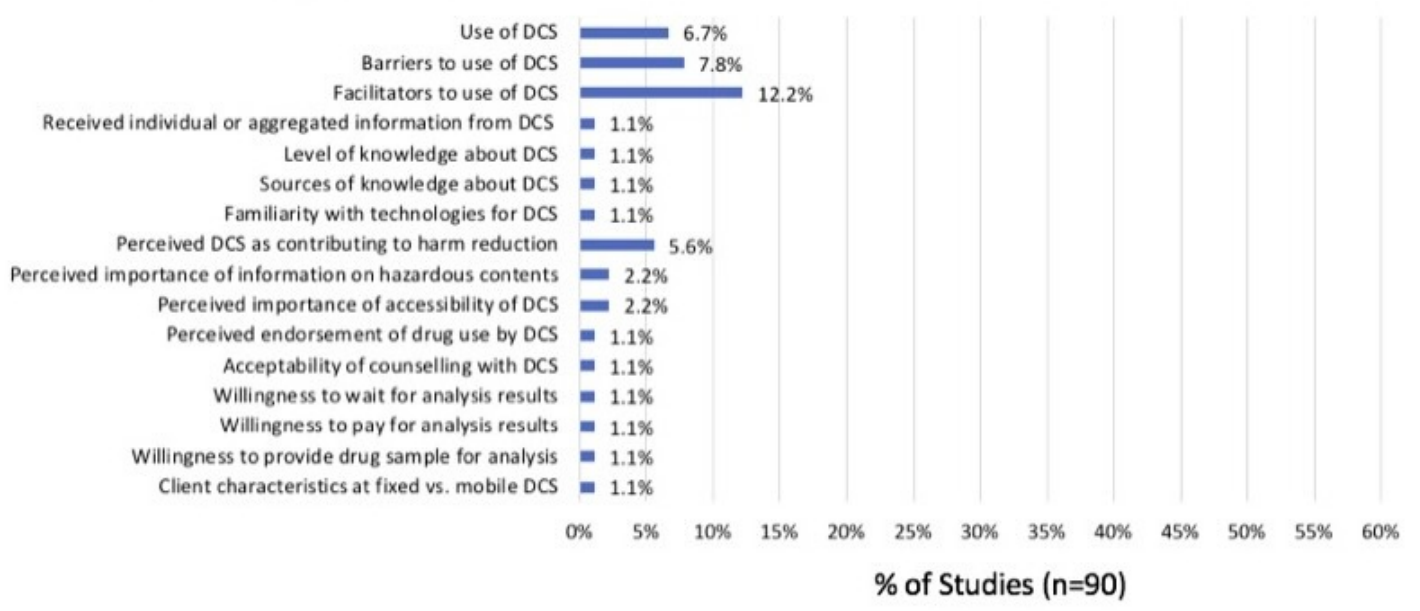

Table 1: Models Described for DCS in Included Studies 


\begin{tabular}{|c|c|c|c|c|c|}
\hline Country & Provider & $\begin{array}{l}\text { culleculü } \\
\text { Model }\end{array}$ & $\begin{array}{l}\text { Mllaiysis } \\
\text { Model }\end{array}$ & Analysis Methods & Population \\
\hline Australia & ACT GTM Pill Testing Pilot & Mobile & Onsite & FTIR & Partygoers \\
\hline Australia & Enlighten & Mobile & $\begin{array}{l}\text { Offsite; } \\
\text { Onsite }\end{array}$ & GC-MS; Reagents & Partygoers \\
\hline Austria & checkit! & Mobile & $\begin{array}{l}\text { Offsite; } \\
\text { Onsite }\end{array}$ & $\begin{array}{l}\text { GC-MS; HP-LC; } \\
\text { LC-DAD; UV } \\
\text { spectroscopy }\end{array}$ & Partygoers \\
\hline Belgium & Modus Fiesta & $\begin{array}{l}\text { Fixed; } \\
\text { Mobile }\end{array}$ & $\begin{array}{l}\text { Offsite; } \\
\text { Onsite }\end{array}$ & GC-MS; Reagents; TLC & Partygoers \\
\hline Canada & $\begin{array}{l}\text { ANKORS (AIDS Network Outreach \& } \\
\text { Support Society) }\end{array}$ & Mobile & Onsite & $\begin{array}{l}\text { Fentanyl test strips; } \\
\text { GC-MS; Raman } \\
\text { spectroscopy; } \\
\text { Reagents }\end{array}$ & Partygoers \\
\hline Canada & $\begin{array}{l}\text { BCCSU (British Columbia Centre on } \\
\text { Substance Use) }\end{array}$ & $\begin{array}{l}\text { Fixed; } \\
\text { Mobile }\end{array}$ & Onsite & $\begin{array}{l}\text { Fentanyl test strips; } \\
\text { FTIR }\end{array}$ & $\begin{array}{l}\text { Structurally vulnerable people } \\
\text { who use/inject drugs; Partygoers }\end{array}$ \\
\hline France & $\begin{array}{l}\text { SINTES (National Identification System } \\
\text { for Drugs and Other Substances) }\end{array}$ & Mobile & Offsite & GC-MS & PWUD \\
\hline Germany & Hanover Drobs & $\begin{array}{l}\text { Fixed; } \\
\text { Mobile }\end{array}$ & Onsite & Reagents & Partygoers \\
\hline Italy & $\begin{array}{l}\text { BAONPS (Be Aware On Night Pleasure } \\
\text { Safety) }\end{array}$ & Mobile & $\begin{array}{l}\text { Offsite; } \\
\text { Onsite }\end{array}$ & $\begin{array}{l}\text { GC-MS; LC-MS; Raman } \\
\text { spectroscopy }\end{array}$ & Partygoers \\
\hline Netherlands & $\begin{array}{l}\text { DIMS (Drug Information and Monitoring } \\
\text { System) }\end{array}$ & Fixed & $\begin{array}{l}\text { Offsite; } \\
\text { Onsite }\end{array}$ & $\begin{array}{l}\text { GC-MS; GC-NPD; } \\
\text { LC-DAD; NMR } \\
\text { spectroscopy; } \\
\text { Reagents; TLC }\end{array}$ & PWUD recreationally \\
\hline Portugal & Check!n & Mobile & Onsite & Reagents; TLC & Partygoers \\
\hline Portugal & Kosmicare Association & Mobile & Onsite & Reagents; TLC & Partygoers \\
\hline Slovenia & DrogArt & Fixed & Offsite & Unspecified & “High-risk" PWUD; Partygoers \\
\hline Spain & Ailaket & Mobile & Unspecified & TLC & PWUD recreationally \\
\hline Spain & Energy Control & $\begin{array}{l}\text { Fixed; } \\
\text { Mobile; } \\
\text { Postal }\end{array}$ & Offsite & $\begin{array}{l}\text { GC-MS; LC-MS; TLC; } \\
\text { UV spectroscopy }\end{array}$ & PWUD recreationally \\
\hline Switzerland & Streetwork & $\begin{array}{l}\text { Fixed; } \\
\text { Mobile }\end{array}$ & Onsite & $\begin{array}{l}\text { GC-MS; HP-LC; } \\
\text { LC-DAD; UV } \\
\text { spectroscopy }\end{array}$ & Partygoers \\
\hline UK & The Loop & Mobile & Onsite & $\begin{array}{l}\text { FTIR; Mass loss } \\
\text { analysis; Reagents }\end{array}$ & Partygoers \\
\hline US & DanceSafe & Mobile & Onsite & Reagents & Partygoers \\
\hline US & EcstasyData & Postal & Offsite & GC-MS; Reagents & $\begin{array}{l}\text { PWUD; Drug sellers; Club owners; } \\
\text { Parents }\end{array}$ \\
\hline US & Organizations in Rhode Island & $\begin{array}{l}\text { Personal } \\
\text { Use }\end{array}$ & Offsite & Fentanyl test strips & Young PWUD \\
\hline US & SAC (Syringe Access Collaborative) & $\begin{array}{l}\text { Fixed; } \\
\text { Personal } \\
\text { Use }\end{array}$ & $\begin{array}{l}\text { Onsite; } \\
\text { Offsite }\end{array}$ & Fentanyl test strips & PWUD \\
\hline US & Syringe services program in Boston & $\begin{array}{l}\text { Personal } \\
\text { Use }\end{array}$ & Offsite & Fentanyl test strips & Street-based PWUD \\
\hline US & $\begin{array}{l}\text { Syringe services programs in New York } \\
\text { City }\end{array}$ & $\begin{array}{l}\text { Personal } \\
\text { Use }\end{array}$ & Offsite & Fentanyl test strips & $\begin{array}{l}\text { People physically dependent on } \\
\text { opioids }\end{array}$ \\
\hline US & Urban Survivors Union & $\begin{array}{l}\text { Personal } \\
\text { Use }\end{array}$ & Offsite & Fentanyl test strips & People who inject drugs \\
\hline
\end{tabular}




\section{Analysis Methods Abbreviations}

FTIR: Fourier transform infrared spectroscopy

GC-MS: Gas chromatography-mass spectrometry

GC-NPD: Gas chromatography nitrogen phosphorous detection

HP-LC: High-performance liquid chromatography

LC-MS: Liquid chromatography-mass spectrometry

LC-DAD: Liquid chromatography with diode array detection

NMR: Nuclear magnetic resonance

TLC: Thin layer chromatography

UV: UltraViolet Visible

Table 2: Characteristics of Included Studies 


\begin{tabular}{|c|c|c|c|c|}
\hline Characteristic & $\begin{array}{l}\text { Total } \\
\text { N (\%) } \\
(\mathbf{n = 9 0 )}\end{array}$ & $\begin{array}{l}\text { Articles } \\
N(\%) \\
(n=54)\end{array}$ & $\begin{array}{l}\text { Conference Abstracts } \\
\text { N (\%) } \\
(n=19)\end{array}$ & $\begin{array}{l}\text { Grey Literature } \\
\text { N (\%) } \\
(n=17)\end{array}$ \\
\hline \multicolumn{5}{|l|}{ Country } \\
\hline Australia & $2(2.2)$ & $1(1.9)$ & $0(0)$ & $1(5.9)$ \\
\hline Austria & $1(1.1)$ & $0(0)$ & $0(0)$ & $1(5.9)$ \\
\hline Belgium & $2(2.2)$ & $0(0)$ & $0(0)$ & 2 (11.8) \\
\hline Canada & $9(10)$ & $7(13)$ & $0(0)$ & $2(11.8)$ \\
\hline Colombia & $1(1.1)$ & $0(0)$ & $0(0)$ & $1(5.9)$ \\
\hline France & $2(2.2)$ & $2(3.7)$ & $0(0)$ & $0(0)$ \\
\hline Italy & $2(2.2)$ & $1(1.9)$ & $0(0)$ & $1(5.9)$ \\
\hline Netherlands & $19(21.1)$ & 16 (29.6) & $0(0)$ & $3(17.6)$ \\
\hline New Zealand & $3(3.3)$ & $0(0)$ & $0(0)$ & $3(17.6)$ \\
\hline Portugal & $3(3.3)$ & $3(5.5)$ & $0(0)$ & $0(0)$ \\
\hline Slovenia & $1(1.1)$ & $1(1.9)$ & $0(0)$ & $0(0)$ \\
\hline Spain & 30 (33.3) & $12(22.2)$ & $18(94.7)$ & $0(0)$ \\
\hline Switzerland & $1(1.1)$ & $1(1.9)$ & $0(0)$ & $0(0)$ \\
\hline UK & $2(2.2)$ & $1(1.9)$ & $0(0)$ & $1(5.9)$ \\
\hline US & $9(10)$ & $7(13)$ & $1(5.3)$ & $1(5.9)$ \\
\hline Multi-country* & $3(3.3)$ & $2(3.7)$ & $0(0)$ & $1(5.9)$ \\
\hline \multicolumn{5}{|l|}{ Domains** } \\
\hline (a) Influence of DCS on behaviour of PWUD & $31(34.4)$ & $14(25.9)$ & $0(0)$ & $17(100)$ \\
\hline (b) Monitoring of drug markets by DCS & $63(70)$ & 44 (81.5) & $19(100)$ & $0(0)$ \\
\hline (c) Outcomes related to models of DCS & $17(18.9)$ & 17 (31.5) & $0(0)$ & $0(0)$ \\
\hline \multicolumn{5}{|l|}{ Study Design } \\
\hline Cross-sectional & $49(54.4)$ & $24(44.4)$ & $11(57.9)$ & $14(82.4)$ \\
\hline Repeated Cross-sectional & 30 (33.3) & 21 (38.9) & $8(42.1)$ & $1(5.9)$ \\
\hline Longitudinal & $1(1.1)$ & $1(1.9)$ & $0(0)$ & $0(0)$ \\
\hline Time-series & $2(2.2)$ & $2(3.7)$ & $0(0)$ & $0(0)$ \\
\hline Qualitative & $7(7.8)$ & $5(9.3)$ & $0(0)$ & $2(11.8)$ \\
\hline Case Report & $1(1.1)$ & $1(1.9)$ & $0(0)$ & $0(0)$ \\
\hline
\end{tabular}

*One North American article including Canada, Mexico, and the US, two multi-country European studies (one article including Austria, Belgium, Netherlands, Portugal, Spain, and Switzerland, and one book including Austria, Netherlands, and Germany).

**Combined total exceeds number of studies because some evaluated more than one domain.

\section{Appendix A: Full Search Strategies and Results}

As run on July 26-August 1, 2018 and subsequently updated October 19, 2019. 
Database: Ovid MEDLINE: Epub Ahead of Print, In-Process \& Other Non-Indexed Citations, Ovid MEDLINE $®$ Daily and Ovid MEDLINE $®<1946-P r e s e n t>$

Search Strategy:

1 drug checking.tw,kf. (24)

2 (drug test* adj2 (check* or information or service*)).tw,kf. (17)

3 (pill adj2 checking).tw,kf. (6)

4 (pill adj2 testing).tw,kf. (28)

5 (drug test* kit or drug test* kits).tw,kf. (5)

6 checkit!.tw,kf. (4)

7 "drug information and monitoring system".tw,kf. (3)

8 Trans European Drug Information.tw,kf. (1)

9 street drug analysis.tw,kf. (8)

10 adulterant screening.tw,kf. (4)

11 dancesafe*.tw,kf. (6)

12 saferparty*.tw,kf. (0)

13 raveitsafe*.tw,kf. (0)

14 sintes.tw,kf. (4)

15 Technoplus.tw,kf. (0)

16 Modus Fiesta.tw,kf. (0)

17 DrogArt.tw,kf. (0)

18 ANKORS Festival Harm Reduction.tw,kf. (0)

19 EcstasyData.tw,kf. (0)

20 drugsdata.tw,kf. (1)

21 Lonja Laket Project.tw,kf. (0)

22 ACT Investigation of Novel Substances Project.tw,kf. (0)

23 Servicio de Analisis de Sustancias.tw,kf. (0)

24 knowyourstuffnz.tw,kf. (0)

25 drug test strips.tw,kf. (0)

26 Kosmicare.tw,kf. (1)

27 Fentanyl Test*.tw,kf. (10)

28 or/1-27 (112)

29 Drug Users/ (2516)

30 Drug Overdose/pc [Prevention \& Control] (791)

31 exp Street Drugs/an (1233)

32 Harm Reduction/ (2454)

33 Needle-Exchange Programs/ (1591) 
34 exp Cocaine/an (1085)

35 Heroin/an (328)

36 exp Narcotics/an (2585)

37 n-methyl-3,4-methylenedioxyamphetamine/an (210)

38 methamphetamine/an (482)

39 Designer Drugs/an (317)

40 exp Amphetamine/an (465)

41 exp Hallucinogens/an (1373)

42 Psychotropic Drugs/an (375)

43 Lysergic Acid Diethylamide/an (142)

44 illicit drug*.tw,kf. (9793)

45 illegal drug*.tw,kf. (2067)

46 safe* injecti* facilit*.tw,kf. (67)

47 safe* injecti* site*.tw,kf. (28)

48 safe injecti* cent*.tw,kf. (0)

49 supervised injecti* site*.tw,kf. (23)

50 supervised injecti* facilit*.tw,kf. (125)

51 supervised injecti* cent*.tw,kf. (29)

52 needle exchange.tw,kf. (921)

53 harm reduction.tw,kf. (3784)

54 (IDU or IDUs).tw,kf. (5266)

55 people who inject drugs.tw,kf. (1584)

56 "people who use drugs".tw,kf. (326)

57 novel psychoactive substance*.tw,kf. (334)

58 new psychoactive substance*.tw,kf. (671)

59 novel psychoactive drug*.tw,kf. (31)

60 ((drug* or narcotic* or substance) adj (user or users or abuse* or addict*)).tw,kf. (67656)

61 or/29-60 (90031)

62 Drug Contamination/ (10295)

63 exp Reagent Kits, Diagnostic/ (19202)

64 drug testing.tw,kf. (3580)

6562 or 63 or 64 (33003)

$66 \quad 61$ and 65 (1012)

6728 or $66(1100)$

68 limit 67 to $y r=" 1990$-Current" (952)

69 limit 68 to (comment or editorial or letter) (27)

7068 not 69 (925) 
71 remove duplicates from 70 (925)

\section{Database: Embase Classic+Embase <1947 to 2018 July 26>}

Search Strategy:

1 drug checking.tw,kw. (32)

2 (drug test* adj2 (check* or information or service*)).tw,kw. (28)

3 (pill adj2 checking).tw,kw. (7)

4 (pill adj2 testing).tw,kw. (29)

5 (drug test* kit or drug test* kits).tw,kw. (8)

6 checkit!.tw,kw. (4)

7 "drug information and monitoring system".tw,kw. (4)

8 Trans European Drug Information.tw,kw. (1)

9 street drug analysis.tw,kw. (18)

10 adulterant screening.tw,kw. (4)

11 dancesafe*.tw,kw. (5)

12 saferparty*.tw,kw. (0)

13 raveitsafe*.tw,kw. (0)

14 sintes.tw,kw. (11)

15 Technoplus.tw,kw. (0)

16 Modus Fiesta.tw,kw. (0)

17 DrogArt.tw,kw. (0)

18 ANKORS Festival Harm Reduction.tw,kw. (0)

19 EcstasyData.tw,kw. (1)

20 drugsdata.tw,kw. (3)

21 Lonja Laket Project.tw,kw. (0)

22 ACT Investigation of Novel Substances Project.tw,kw. (0)

23 Servicio de Analisis de Sustancias.tw,kw. (0)

24 knowyourstuffnz.tw,kw. (0)

25 drug test strips.tw,kw. (0)

26 Kosmicare.tw,kw. (1)

27 Fentanyl Test*.tw,kw. (16)

281 or 2 or 3 or 4 or 5 or 6 or 7 or 8 or 9 or 10 or 11 or 12 or 13 or 14 or 15 or 16 or 17 or 18 or 19 or 20

or 21 or 22 or 23 or 24 or 25 or 26 or 27 (161)

29 drug overdose/pc [Prevention] (428)

30 street drug/an [Drug Analysis] (277)

31 harm reduction/ (4799) 
32 designer drug/an (153)

33 illicit drug/an (85)

34 diamorphine/an (278)

35 cocaine/an (853)

36 exp methamphetamine/an (664)

37 exp psychedelic agent/an (2323)

38 exp fentanyl derivative/an (541)

39 illicit drug*.tw,kw. (13448)

40 illegal drug*.tw,kw. (2788)

41 safe* injecti* facilit*.tw,kw. (80)

42 safe* injecti* site*.tw,kw. (36)

43 safe injecti* cent*.tw,kw. (0)

44 supervised injecti* site*.tw,kw. (28)

45 supervised injecti* facilit*.tw,kw. (150)

46 supervised injecti* cent*.tw,kw. (44)

47 needle exchange.tw,kw. (1097)

48 harm reduction.tw,kw. (5026)

49 (IDU or IDUs).tw,kw. (7156)

50 people who inject drugs.tw,kw. (1972)

51 "people who use drugs".tw,kw. (418)

52 novel psychoactive substance*.tw,kw. (511)

53 new psychoactive substance*.tw,kw. (826)

54 novel psychoactive drug*.tw,kw. (40)

55 ((drug* or narcotic* or substance) adj (user or users or abuse* or addict*)).tw,kw. (92145)

56 or/29-55 (118255)

57 test strip/ (3692)

58 drug contamination/ (8141)

59 drug testing.tw,kw. (5008)

60 drug analysis/ (92023)

61 drug discrimination/ (2882)

62 drug identification/ (56631)

6357 or 58 or 59 or 60 or 61 or $62(135582)$

6456 and 63 (1606)

6528 or 64 (1741)

66 limit 65 to $y r=" 1990$-Current" (1486)

67 limit 66 to (editorial or letter) (20)

6866 not 67 (1466) 
69 limit 68 to embase (1143)

70 remove duplicates from 69 (1126)

Ovid Database: EBM Reviews - Cochrane Central Register of Controlled Trials <June 2018>, EBM Reviews - Cochrane Database of Systematic Reviews <2005 to July 18, 2018> Search Strategy:

1 drug checking.tw,kw. (1)

2 (drug test* adj2 (check* or information or service*)).tw,kw. (3)

3 (pill adj2 checking).tw,kw. (1)

4 (pill adj2 testing).tw,kw. (6)

5 (drug test* kit or drug test* kits).tw,kw. (1)

6 checkit!.tw,kw. (0)

7 "drug information and monitoring system".tw,kw. (0)

8 Trans European Drug Information.tw,kw. (0)

9 street drug analysis.tw,kw. (0)

10 adulterant screening.tw,kw. (0)

11 dancesafe*.tw,kw. (0)

12 saferparty*.tw,kw. (0)

13 raveitsafe*.tw,kw. (0)

14 sintes.tw,kw. (2)

15 Technoplus.tw,kw. (0)

16 Modus Fiesta.tw,kw. (0)

17 DrogArt.tw,kw. (0)

18 ANKORS Festival Harm Reduction.tw,kw. (0)

19 EcstasyData.tw,kw. (0)

20 drugsdata.tw,kw. (1)

21 Lonja Laket Project.tw,kw. (0)

22 ACT Investigation of Novel Substances Project.tw,kw. (0)

23 Servicio de Analisis de Sustancias.tw,kw. (0)

24 knowyourstuffnz.tw,kw. (0)

25 drug test strips.tw,kw. (0)

26 Kosmicare.tw,kw. (0)

27 Fentanyl Test*.tw,kw. (5)

281 or 2 or 3 or 4 or 5 or 6 or 7 or 8 or 9 or 10 or 11 or 12 or 13 or 14 or 15 or 16 or 17 or 18 or 19 or 20 or 21 or 22 or 23 or 24 or 25 or 26 or $27(20)$

29 Drug Contamination/ (55) 
30 exp Reagent Kits, Diagnostic/ (261)

31 drug testing.tw,kw. (185)

3229 or 30 or 31 (500)

33 Drug Users/ (100)

34 Drug Overdose/pc (2)

35 exp Street Drugs/an (0)

36 Harm Reduction/ (98)

37 Needle-Exchange Programs/ (36)

38 exp Cocaine/an (0)

39 Heroin/an (0)

40 exp Narcotics/an (0)

41 n-methyl-3,4-methylenedioxyamphetamine/an (0)

42 methamphetamine/an (0)

43 Designer Drugs/an (0)

44 exp Amphetamine/an (0)

45 exp Hallucinogens/an (2)

46 Psychotropic Drugs/an (0)

47 Lysergic Acid Diethylamide/an (0)

48 illicit drug*.tw,kw. (700)

49 illegal drug*.tw,kw. (82)

50 safe* injecti* facilit*.tw,kw. (2)

51 safe* injecti* site*.tw,kw. (21)

52 safe injecti* cent*.tw,kw. (0)

53 supervised injecti* site*.tw,kw. (0)

54 supervised injecti* facilit*.tw,kw. (0)

55 supervised injecti* cent*.tw,kw. (0)

56 needle exchange.tw,kw. (44)

57 harm reduction.tw,kw. (239)

58 (IDU or IDUs).tw,kw. (282)

59 people who inject drugs.tw,kw. (92)

60 "people who use drugs".tw,kw. (18)

61 novel psychoactive substance*.tw,kw. (11)

62 new psychoactive substance*.tw,kw. (7)

63 novel psychoactive drug*.tw,kw. (0)

64 ((drug* or narcotic* or substance) adj (user or users or abuse* or addict*)).tw,kw. (5112)

65 or/33-64 (6098)

$66 \quad 32$ and 65 (46) 
6728 or $66(62)$

68 remove duplicates from 67 (62)

69 limit 68 to $y r=" 1990$-Current" (61)

\section{Database: PsyclNFO <1806 to July Week 4 2018>}

Search Strategy:

1 drug checking.tw,kw. (19)

2 (drug test* adj2 (check* or information or service*)).tw,kw. (7)

3 (pill adj2 checking).tw,kw. (5)

4 (pill adj2 testing).tw,kw. (13)

5 (drug test* kit or drug test* kits).tw,kw. (2)

6 checkit!.tw,kw. (0)

7 "drug information and monitoring system".tw,kw. (2)

8 Trans European Drug Information.tw,kw. (0)

9 street drug analysis.tw,kw. (6)

10 adulterant screening.tw,kw. (0)

11 dancesafe*.tw,kw. (4)

12 saferparty*.tw,kw. (0)

13 raveitsafe*.tw,kw. (0)

14 sintes.tw,kw. (2)

15 Technoplus.tw,kw. (0)

16 Modus Fiesta.tw,kw. (0)

17 DrogArt.tw,kw. (0)

18 ANKORS Festival Harm Reduction.tw,kw. (0)

19 EcstasyData.tw,kw. (2)

20 drugsdata.tw,kw. (0)

21 Lonja Laket Project.tw,kw. (0)

22 ACT Investigation of Novel Substances Project.tw,kw. (0)

23 Servicio de Analisis de Sustancias.tw,kw. (0)

24 knowyourstuffnz.tw,kw. (0)

25 drug test strips.tw,kw. (0)

26 Kosmicare.tw,kw. (0)

27 Fentanyl Test*.tw,kw. (2)

281 or 2 or 3 or 4 or 5 or 6 or 7 or 8 or 9 or 10 or 11 or 12 or 13 or 14 or 15 or 16 or 17 or 18 or 19 or 20 or 21 or 22 or 23 or 24 or 25 or 26 or 27 (53)

29 illicit drug*.tw,kw. (6979) 
30 illegal drug*.tw,kw. (1788)

31 safe* injecti* facilit*.tw,kw. (44)

32 safe* injecti* site*.tw,kw. (9)

33 safe injecti* cent*.tw,kw. (1)

34 supervised injecti* site*.tw,kw. (16)

35 supervised injecti* facilit*.tw,kw. (97)

36 supervised injecti* cent*.tw,kw. (27)

37 needle exchange.tw,kw. (486)

38 harm reduction.tw,kw. (3596)

39 (IDU or IDUs).tw,kw. (2126)

40 people who inject drugs.tw,kw. (802)

41 "people who use drugs".tw,kw. (217)

42 novel psychoactive substance*.tw,kw. (138)

43 new psychoactive substance*.tw,kw. (186)

44 novel psychoactive drug*.tw,kw. (18)

45 ((drug* or narcotic* or substance) adj (user or users or abuse* or addict*)).tw,kw. (64687)

46 intravenous drug usage/ or exp drug abuse/ or exp drug addiction/ or needle exchange programs/ (107979)

47 exp drug abuse/ or polydrug abuse/ (105154)

48 intravenous drug usage/ or "substance use disorder"/ or drug overdoses/ (10743)

49 exp harm reduction/ (3221)

50 exp narcotic drugs/ (27313)

51 cocaine/ (12911)

52 exp amphetamine/ (11190)

53 designer drugs/ or exp hallucinogenic drugs/ (3724)

54 or/29-53 (175995)

55 drug testing.tw. (795)

5654 and $55(411)$

5728 or 56 (456)

58 limit 57 to $y r=" 1990$-Current" (418)

59 limit 58 to (chapter or "column/opinion" or "comment/reply" or editorial or letter or review-book or review-media or review-software \& other) (82)

6058 not 59 (336)

61 remove duplicates from 60 (336)

\section{Scopus}

Search Strategy: 
Limited to 1990-present

( TITLE-ABS-KEY ( kosmicare OR "drug test strip*" OR knowyourstuffnz OR "Servicio de Analisis de Sustancias" OR "ACT Investigation of Novel Substances Project" OR "Lonja Laket Project" OR drugsdata OR ecstasydata )) OR ( TITLE-ABS-KEY (drogart OR "Modus Fiesta" OR technoplus OR sintes OR raveitsafe* OR saferparty* OR dancesafe* OR "adulterant screening" OR "street drug test*" OR "street drug analysis" ) ) OR ( TITLE-ABS-KEY ( "Trans European Drug Information" OR "drug information and monitoring system" OR checkit! OR "drug test* kits" OR "drug test* kit" OR "pill testing" OR "pill checking" OR "drug test* check*" )) OR ( TITLE-ABS-KEY ( "drug test* information" OR "drug test* service" OR "drug checking" ) ) OR ( ( TITLE ( "drug testing" ) AND TITLE-ABS-KEY ( "supervised injecti*" )) ) OR ( ( TITLE ( "drug testing" ) AND TITLE-ABS-KEY ( "street drugs" )) ) OR ( ( TITLE ( "drug testing" ) AND TITLE-ABS-KEY ( "who use drugs" ) )) OR ( ( TITLE ( "drug testing" ) AND TITLE-ABS-KEY ( "who inject drugs" ) ) ) OR ( ( TITLE-ABS-KEY ( "drug testing" ) AND TITLE-ABS-KEY ( "drug overdose" )) ) OR ( ( TITLE ("drug testing" ) AND TITLE-ABS-KEY ("drug users" )))

\section{Web of Science Core Collection: Citation Indexes}

- Science Citation Index Expanded (SCI-EXPANDED) --1900-present

- Social Sciences Citation Index (SSCI) --1956-present

- Conference Proceedings Citation Index- Science (CPCI-S) --1990-present

- Conference Proceedings Citation Index- Social Science \& Humanities (CPCI-SSH) --1990present

- Emerging Sources Citation Index (ESCI) --2015-present

Search Strategy:

Limited to 1990-present

\# 27

205

\#26 OR \#25 OR \#24 OR \#23 OR \#22 OR \#21 OR \#20 OR \#19 OR \#18 OR \#17 OR \#16 OR \#15 OR \#14 OR \#13 OR \#12 OR \#11 OR \#10 OR \#9 OR \#8 OR \#7 OR \#6 OR \#5 OR \#4 OR \#3 OR \#2 OR \#1

\# 26

11

TITLE: ("drug testing") AND TOPIC: (("drug use*"))

\# 25 
1

TITLE: ("drug testing") AND TOPIC: (("harm reduction"))

\# 24

0

TITLE: ("drug testing") AND TOPIC: (("harm minimisation"))

\# 23

0

TITLE: ("drug testing") AND TOPIC: (("harm minimization"))

\# 22

1

TITLE: ("drug testing") AND TOPIC: (("drug abuse"))

\# 21

0

TITLE: ("drug testing") AND TOPIC: (("supervised injection"))

\# 20

0

TITLE: ("drug testing") AND TOPIC: (("safe injection"))

\# 19

2

TITLE: ("drug testing") AND TOPIC: ((fentanyl))

\# 18

3

TITLE: ("drug testing") AND TOPIC: ((overdose))

\# 17

0

TITLE: ("drug testing") AND TOPIC: (("street drug*"))

\# 16

7 
TITLE: ("drug testing") AND TOPIC: (("substance use*"))

\# 15

5

TITLE: ("drug testing") AND TOPIC: (("substance abuse"))

\# 14

5

TITLE: ("drug testing") AND TOPIC: (("illicit drugs"))

\# 13

14

TOPIC: ("drug testing") AND TOPIC: (("drug abuse"))

\# 12

1

TITLE: ("drug testing") AND TOPIC: (("drug abuse"))

\# 11

0

TITLE: ("drug testing") AND TOPIC: (("supervised injection"))

\# 10

0

TITLE: ("drug testing") AND TOPIC: (("safe injection"))

\# 9

35

TS $=($ "drug testing") AND TS $=(($ rave or raves or festival* or concert* or event* or gathering or dance* $))$

\# 8

11

TOPIC: ("drug testing") AND TOPIC: ("drug users")

\# 7

0

TITLE: ("drug testing") AND TOPIC: ("drug overdose") 


\section{\# 6}

1

TITLE: ("drug testing") AND TOPIC: ("who inject drugs")

\# 5

0

TITLE: ("drug testing") AND TOPIC: ("who use drugs")

\# 4

0

TITLE: ("drug testing") AND TOPIC: ("street drugs")

\# 3

0

TITLE: ("drug testing") AND TOPIC: ("supervised injecti*")

\# 2

127

TITLE: ("drug testing")

\# 1

37

TOPIC: (kosmicare OR "drug test strip*" OR knowyourstuffnz OR "Servicio de Analisis de Sustancias" OR "ACT Investigation of Novel Substances Project" OR "Lonja Laket Project" OR drugsdata OR ecstasydata OR drogart OR "Modus Fiesta" OR technoplus OR sintes OR raveitsafe* OR saferparty* OR dancesafe* OR "adulterant screening" OR "street drug test*" OR "street drug analysis" OR "Trans European Drug Information" OR "drug information and monitoring system" OR checkit! OR "drug test* kits" OR "drug test* kit" OR "pill testing" OR "pill checking" OR "drug test* check*" OR "drug test* information" OR "drug test* service" OR "drug checking")

Indexes=SCI-EXPANDED, SSCI, CPCI-S, CPCI-SSH, ESCI Timespan=All years

\section{Dissertations \& Theses Global}

Search Strategy:

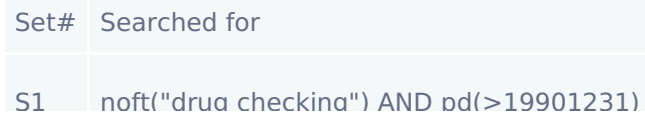


noft("pill testing") AND pd(>19901231)

S11

S15 noft(checkit) AND pd(>19901231)

noft("street drug analysis") AND pd(>19901231)

noft("street drug test*") AND pd(>19901231)

noft(dancesafe*) AND pd(>19901231)

noft(saferparty*) AND pd(>19901231)

noft(raveitsafe*) AND pd(>19901231)

noft(Technoplus) AND pd(>19901231)

noft(DrogArt) AND pd(>19901231)

noft("Modus Fiesta") AND pd(>19901231)

$\mathrm{S} 28$ noft(ANKORS Festival Harm Reduction) AND pd(>19901231) noft(EcstasyData) AND pd(>19901231)

S30 noft(drugsdata) AND pd(>19901231)

S31 noft(Lonja Laket Project) AND pd(>19901231)

S32 noft("ACT Investigation of Novel Substances Project") AND pd(>19901231)

S34 noft("Servicio de Analisis de Sustancias") AND pd(>19901231)

S35 noft(knowyourstuffnz) AND pd(>19901231)
Theses Global

ProQuest Dissertations \&

Theses Global

ProQuest Dissertations \&

Theses Global

ProQuest Dissertations \&

Theses Global

ProQuest Dissertations \& Theses Global

ProQuest Dissertations \& Theses Global

ProQuest Dissertations \& Theses Global

ProQuest Dissertations \& Theses Global

ProQuest Dissertations \& Theses Global

ProQuest Dissertations \& Theses Global

ProQuest Dissertations \& Theses Global

ProQuest Dissertations \& Theses Global

ProQuest Dissertations \& Theses Global

ProQuest Dissertations \& Theses Global

ProQuest Dissertations \& Theses Global

ProQuest Dissertations \& Theses Global

ProQuest Dissertations \& Theses Global

ProQuest Dissertations \& Theses Global

ProQuest Dissertations \& Theses Global

ProQuest Dissertations \& Theses Global

ProQuest Dissertations \& Theses Global

ProQuest Dissertations \& Theses Global

ProQuest Dissertations \& Theses Global

ProQuest Dissertations \& Theses Global

ProQuest Dissertations \& Theses Global

ProQuest Dissertations \& Theses Global 


\section{Database}

Medline (OVID) Results

Embase (OVID)

Cochrane Central Register of Controlled Trials and Cochrane Database of Systematic Reviews (OVID, searched simultaneously)

Web of Science: (searched simultaneously)

- Science Citation Index

- Social Sciences Citation Index (SSCI)

- Conference Proceedings Citation Index- Science (CPCI-S)

- Conference Proceedings Citation Index- Social Science \& Humanities (CPCI-SSH)

- Emerging Sources Citation Index (ESCI)

Dissertations \& Theses Global (ProQuest)

\section{Appendix B: Quality Assessment Forms}

\section{Quality Assessment Tool for Observational Cohort and Cross-Sectional Studies}

Adapted from: Quality Assessment Tool for Observational Cohort and Cross-Sectional Studies. National Heart, Lung, and Blood Institute, 2014.

1. Was the research question or objective in this paper clearly stated?

Yes

No

\section{Cannot Determine}

Not Applicable

2. Was the study population clearly specified and defined?

Yes

No 


\section{Cannot Determine}

Not Applicable

3. Was the participation rate of eligible persons at least $50 \%$ ?

Yes

No

Cannot Determine

Not Applicable

4. Were all the subjects selected or recruited from the same or similar populations (including the same time period)? Were inclusion and exclusion criteria for being in the study prespecified and applied uniformly to all participants? Was the sample representative of the population from which they were recruited?

Yes

No

Cannot Determine

Not Applicable

5. Was a sample size justification, power description, or variance and effect estimates provided?

Yes

No

Cannot Determine

Not Applicable

6. For the analyses in this paper, were the exposure(s) of interest measured prior to the outcome(s) being measured?

Yes

No

Cannot Determine

Not Applicable

7. Was the timeframe sufficient so that one could reasonably expect to see an association between exposure and outcome if it existed?

Yes

No

Cannot Determine

Not Applicable 
8. For exposures that can vary in amount or level, did the study examine different levels of the exposure as related to the outcome (e.g., categories of exposure, or exposure measured as continuous variable)? Yes

No

Cannot Determine

Not Applicable

9. Were the exposure measures (independent variables) clearly defined, valid, reliable, and implemented consistently across all study participants?

Yes

No

Cannot Determine

Not Applicable

10. Was the exposure(s) assessed more than once over time?

Yes

No

Cannot Determine

Not Applicable

11. Were the outcome measures (dependent variables) clearly defined, valid, reliable, and implemented consistently across all study participants?

Yes

No

Cannot Determine

Not Applicable

12. Were the outcome assessors blinded to the exposure status of participants?

Yes

No

Cannot Determine

Not Applicable

13. Was loss to follow-up after baseline $20 \%$ or less?

Yes

No 
Cannot Determine

Not Applicable

14. Were key potential confounding variables measured and adjusted statistically for their impact on the relationship between exposure(s) and outcome(s)

Yes

No

Cannot Determine

Not Applicable

Quality Assessment Tool for Before-After (Pre-Post) Studies with No Control Group

Adapted from: Quality Assessment Tool for Before-After (Pre-Post) Studies with No Control Group. National Heart, Lung, and Blood Institute, 2014.

1. Was the study question or objective clearly stated?

Yes

No

Cannot Determine

Not Applicable

2. Were eligibility/selection criteria for the study population prespecified and clearly described?

Yes

No

Cannot Determine

Not Applicable

3. Were the participants in the study representative of those who would be eligible for the test/service/intervention in the general or clinical population of interest?

Yes

No

Cannot Determine

Not Applicable

4. Were all eligible participants that met the prespecified entry criteria enrolled?

Yes

No

Cannot Determine 
Not Applicable

5. Was the sample size sufficiently large to provide confidence in the findings?

Yes

No

Cannot Determine

Not Applicable

6. Was the test/service/intervention clearly described and delivered consistently across the study population?

Yes

No

Cannot Determine

Not Applicable

7. Were the outcome measures prespecified, clearly defined, valid, reliable, and assessed consistently across all study participants?

Yes

No

Cannot Determine

Not Applicable

8. Were the people assessing the outcomes blinded to the participants' exposures/interventions?

Yes

No

Cannot Determine

Not Applicable

9. Was the loss to follow-up after baseline $20 \%$ or less? Were those lost to follow-up accounted for in the analysis?

Yes

No

Cannot Determine

Not Applicable

10. Did the statistical methods examine changes in outcome measures from before to after the intervention? Were statistical tests done that provided $p$ values for the pre-to-post changes? 
Yes

No

Cannot Determine

Not Applicable

11. Were outcome measures of interest taken multiple times before the intervention and multiple times after the intervention (i.e., did they use an interrupted time-series design)?

Yes

No

Cannot Determine

Not Applicable

12. If the intervention was conducted at a group level (e.g., a whole hospital, a community, etc.) did the statistical analysis take into account the use of individual-level data to determine effects at the group level?

Yes

No

Cannot Determine

Not Applicable

\section{Appendix C: Included Studies}

1. 2018/2019 Results. KnowYourStuffNZ; 2019.

2. $2017 / 2018$ Results. KnowYourStuffNZ; 2018.

3. 2016/2017 Results. KnowYourStuffNZ; 2017.

4. Angelats M, Galindo L, Grifell M, Palma A, Martínez L, Pujol L, et al. PCP analogues in samples of Barcelona from 2009 to 2015. European Psychiatry. 2016;33(S1):S117.

5. Bardwell G, Boyd J, Arredondo J, McNeil R, Kerr T. Trusting the source: the potential role of drug dealers in reducing drug-related harms via drug checking. Drug and Alcohol Dependence. 2019;198:1-6.

6. Bardwell G, Boyd J, Tupper K, Kerr T. "We don't got that kind of time, man. We're trying to get high!": Exploring potential use of drug checking technologies among structurally vulnerable people who use drugs. International Journal of Drug Policy. 2019;71:125-32.

7. Benschop A, Rabes M, Korf D. Pill Testing, Ecstasy \& Prevention: A Scientific Evaluation in Three European Cities: Rozenberg Publishers; 2002.

8. Blanckaert P, van Amsterdam J, Brunt T, van den Berg J, Van Durme F, Maudens K, et al. 4-Methylamphetamine: a health threat for recreational amphetamine users. Journal of Psychopharmacology. 2013;27(9):817-22. 
9. Bossong M, Brunt T, Van Dijk J, Rigter S, Hoek J, Goldschmidt H, et al. mCPP: an undesired addition to the ecstasy market. Journal of Psychopharmacology. 2010;24(9):1395-401.

10. Brunt T, Atkinson A, Nefau T, Martinez M, Lahaie E, Malzcewski A, et al. Online test purchased new psychoactive substances in 5 different European countries: a snapshot study of chemical composition and price. International Journal of Drug Policy. 2017;44:105-14.

11. Brunt T, Nagy C, Bücheli A, Martins D, Ugarte M, Beduwe C, et al. Drug testing in Europe: monitoring results of the Trans European Drug Information (TEDI) project. Drug Testing and Analysis. 2017;9(2):18898.

12. Brunt $T$, Koeter M, Niesink R, van den Brink W. Linking the pharmacological content of ecstasy tablets to the subjective experiences of drug users. Psychopharmacology. 2012;220(4):751-62.

13. Brunt $T$, Niesink R, van den Brink W. Impact of a transient instability of the ecstasy market on health concerns and drug use patterns in The Netherlands. International Journal of Drug Policy. 2012;23(2):13440.

14. Brunt T, Niesink R. The Drug Information and Monitoring System (DIMS) in the Netherlands: implementation, results, and international comparison. Drug Testing and Analysis. 2011;3(9):621-34.

15. Brunt T, Poortman A, Niesink R, van den Brink W. Instability of the ecstasy market and a new kid on the block: mephedrone. Journal of Psychopharmacology. 2011;25(11):1543-7.

16. Brunt T, van Laar M, Niesink R, van den Brink W. The relationship of quality and price of the psychostimulants cocaine and amphetamine with health care outcomes. Drug and Alcohol Dependence. 2010;111(1-2):21-9.

17. Brunt T, Rigter S, Hoek J, Vogels N, van Dijk P, Niesink R. An analysis of cocaine powder in the Netherlands: content and health hazards due to adulterants. Addiction. 2009;104(5):798-805.

18. Camilleri A, Caldicott D. Underground pill testing, down under. Forensic Science International. 2005;151(1):53-8.

19. Caudevilla F, Ventura M, Fornís I, Barratt M, Vidal C, Quintana P, et al. Results of an international drug testing service for cryptomarket users. International Journal of Drug Policy. 2016;35:38-41.

20. Caudevilla F, Ventura M, Indave B, Fornís I. Presence and composition of cathinone derivatives in drug samples taken from a drug test service in Spain (2010-2012). Human Psychopharmacology: Clinical and Experimental. 2013;28(4):341-4.

21. Caudevilla F, Riba J, Ventura M, González D, Farré M, Barbanoj M, et al. 4-Bromo-2, 5dimethoxyphenethylamine (2C-B): presence in the recreational drug market in Spain, pattern of use and subjective effects. Journal of Psychopharmacology. 2012;26(7):1026-35.

22. de Dios M, Monteagudo E, Trabsa A, Grifell M, Galindo L, Quintana P, et al. Ethylone: a synthetic cathinone emerging in Barcelona. European Psychiatry. 2017;41(S1):S860.

23. Delile J-M, Gachie J-P. Ecstasy et réduction des risques: La place du testing et des analyses de substances. Alcoologie et Addictologie. 2002;24(4):311-8.

24. Dinamarca F, Grifell M, Palma A, Ventura M, Fornís I, Galindo L, et al. Benzodiazepines as novel 
psychoactive substances (NPS): characterization of the use in a sample of subjects asking for counselling. European Neuropsychopharmacology. 2016;2(26):S699.

25. Drug safety testing at festivals and night clubs. Royal Society for Public Health; 2017.

26. Ezquiaga I, Grifell M, Galindo L, Martínez L, Palma A, Quintana P, et al. 25I-NBOMe: the legal LSD. European Psychiatry. 2016;33(S1):S72-S3.

27. Fabregat-Safont D, Carbón X, Ventura M, Fornís I, Hernández F, Ibáñez M. Characterization of a recently detected halogenated aminorex derivative: para-fluoro-4-methylaminorex (4' F-4-MAR). Scientific Reports. 2019;9(1):1-8.

28. Fabregat-Safont D, Carbón X, Gil C, Ventura M, Sancho J, Hernández F, et al. Reporting the novel synthetic cathinone 5-PPDI through its analytical characterization by mass spectrometry and nuclear magnetic resonance. Forensic Toxicology. 2018;36(2):447-57.

29. Fabregat-Safont D, Carbón X, Ventura M, Fornís I, Guillamón E, Sancho J, et al. Updating the list of known opioids through identification and characterization of the new opioid derivative 3, 4-dichloro- $\mathrm{N}$-(2(diethylamino) cyclohexyl)-N-methylbenzamide (U-49900). Scientific Reports. 2017;7(1):1-14. 30. Fabregat-Safont D, Fornís I, Ventura M, Gil C, Calzada N, Sancho J, et al. Identification and characterization of a putative new psychoactive substance, 2-(2-(4-chlorophenyl) acetamido)-3methylbutanamide, in Spain. Drug Testing and Analysis. 2017;10.

31. Fentanyl Test Strip Pilot: San Francisco August 2017 - January 2018. Harm Reduction Coalition; 2018. 32. Galindo L, Grifell M, Quintana P, Palma A, Tirado J, Ventura M, et al. The synthetic cannabinoids: JWH, four years of analysis. European Psychiatry. 2016;33(S1):S115-S6.

33. Galindo L, Quintana P, Grifell M, Palma A, Gil C, Fornís I, et al. Evolution of the composition of cannabis in Spain. European Neuropsychopharmacology. 2016;26:S682.

34. Gerace E, Seganti F, Luciano C, Lombardo T, Di Corcia D, Teifel H, et al. On-site identification of psychoactive drugs by portable Raman spectroscopy during drug-checking service in electronic music events. Drug and Alcohol Review. 2019;38(1):50-6.

35. Giraudon I, Bello P-Y. Monitoring ecstasy content in France: results from the National Surveillance System 1999-2004. Substance Use \& Misuse. 2007;42(10):1567-78.

36. Goldman J, Waye K, Periera K, Krieger M, Yedinak J, Marshall B. Perspectives on rapid fentanyl test strips as a harm reduction practice among young adults who use drugs: a qualitative study. Harm Reduction Journal. 2019;16(3):1-11.

37. Grifell M, Galindo L, Ventura M, Ribera E, Quintana P, Palma A, et al. The cat and the mouse game: is there a shift towards more dangerous substances in the cathinone illicit market? European Psychiatry. 2017;41(S1):S863.

38. Grifell M, Ventura M, Carbón X, Quintana P, Galindo L, Palma A, et al. Patterns of use and toxicity of new para-halogenated substituted cathinones: 4-CMC (clephedrone), 4-CEC (4-chloroethcatinone) and 4BMC (brephedrone). Human Psychopharmacology: Clinical and Experimental. 2017;32:E2621.

39. Grifell M, Quintana P, Torrens M, Palma A, Galindo L, Ventura M, et al. "Flakka": an emerging trend or a 
mass media phenomenon? Presence of alpha-PVP in samples submitted to a drug checking facility. European Psychiatry. 2016;33(S1):S442.

40. Grifell M, Palma A, Ventura M, Fornís I, Dinamarca F, Torrens M, et al. A trip to the unknown: 2, 5Dimethoxy-4-chloroamphetamine (DOC) sold as LSD: study on samples delivered by users asking for substance analysis. European Neuropsychopharmacology. 2015;25:S620.

41. Hondebrink L, Nugteren-van Lonkhuyzen J, Van Der Gouwe D, Brunt T. Monitoring new psychoactive substances (NPS) in The Netherlands: data from the drug market and the Poisons Information Centre. Drug and Alcohol Dependence. 2015;147:109-15.

42. Houioux G, Favresse D, De Smet P, Piette D. Réduction des risques avec analyse de nouvelles drogues de synthèse: Evaluation. 2006.

43. Huberty C, Favresse D. Evaluation des actions de réduction des risques dans le cadre des activités de testing menées par l'ASBL Modus Vivendi: Rapport de recherche. 2010.

44. Hungerbuehler I, Buecheli A, Schaub M. Drug checking: a prevention measure for a heterogeneous group with high consumption frequency and polydrug use-evaluation of Zurich's drug checking services. Harm Reduction Journal. 2011;8(16):1-6.

45. Italian Results. BAONPS (Be Aware On Night Pleasure Safety); 2014.

46. Karamouzian M, Dohoo C, Forsting S, McNeil R, Kerr T, Lysyshyn M. Evaluation of a fentanyl drug checking service for clients of a supervised injection facility, Vancouver, Canada. Harm Reduction Journal. 2018;15(46):1-8

47. Koeter M. Laat je xtc testen!: Evaluatie van de testservice van de Brijder Stichting: Amsterdam Institute for Addiction Research; 1997.

48. Korf D, Benschop A, Brunt T. Pillen testen in Nederland: Een onderzoek naar versterking van de monitor uitgaansdrugs: Rozenberg Publishers; 2003.

49. Krieger M, Goedel W, Buxton J, Lysyshyn M, Bernstein E, Sherman S, et al. Use of rapid fentanyl test strips among young adults who use drugs. International Journal of Drug Policy. 2018;61:52-8.

50. Kriener H, Schmid R. Check your pills. Check your life. ChEck iT! High quality on-site testing of illicit substances. Information, counselling and safer use measures at raves in Austria. 2005.

51. Linsen F, Koning R, van Laar M, Niesink R, Koeter M, Brunt T. 4-Fluoroamphetamine in the Netherlands: more than a one-night stand. Addiction. 2015;110(7):1138-43.

52. Makkai T, Macleod M, Vumbaca G, Hill P, Caldicott D, Noffs M, et al. Report on Canberra GTM Harm Reduction Service. Harm Reduction Australia; 2018.

53. Martínez L, Grifell M, Galindo L, Ezquiaga I, Quintana P, Ventura M, et al. Methylone consumption characterized through samples handled by users. European Psychiatry. 2016;33(S1):S117.

54. Martins D, Barratt M, Pires C, Carvalho H, Ventura M, Fornís I, et al. The detection and prevention of unintentional consumption of DOx and 25x-NBOMe at Portugal's Boom Festival. Human Psychopharmacology: Clinical and Experimental. 2017;32:E2608.

55. Martins D, Valente H, Pires C. CHECK!NG: the last frontier for Harm Reduction in party settings. Saúde 
e Sociedade. 2015;24(2):646-60.

56. McCrae K, Tobias S, Tupper K, Arredondo J, Henry B, Mema S, et al. Drug checking services at music festivals and events in a Canadian setting. Drug and Alcohol Dependence. 2019;205:107589.

57. McKnight C, Des Jarlais D. Being "hooked up" during a sharp increase in the availability of illicitly manufactured fentanyl: adaptations of drug using practices among people who use drugs (PWUD) in New York City. International Journal of Drug Policy. 2018;60:82-8.

58. Measham F. Drug safety testing, disposals and dealing in an English field: exploring the operational and behavioural outcomes of the UK's first onsite 'drug checking'service. International Journal of Drug Policy. 2019;67:102-7.

59. Mema S, Sage C, Xu Y, Tupper K, Ziemianowicz D, McCrae K, et al. Drug checking at an electronic dance music festival during the public health overdose emergency in British Columbia. Canadian Journal of Public Health. 2018;109:740-4.

60. Michelow W, Dowden C. "Start Small, Take it Easy": Results from the ANKORS Harm Reduction Survey at the 2013 Shambhala Music Festival. ANKORS (AIDS Network Outreach \& Support Society); 2015.

61. Monteagudo E, de Dios M, Trabsa A, Grifell M, Galindo L, Quintana P, et al. Is methylone a new public health threat in Spain? European Psychiatry. 2017;41(S1):S871.

62. Morris V, Gordillo C. Informe de Resultados Encuesta de Satisfacción y Cambio de Comportamiento Échele Cabeza 2016. Échele Cabeza; 2016.

63. Munn M, Lund A, Golby R, Turris S. Observed benefits to on-site medical services during an annual 5day electronic dance music event with harm reduction services. Prehospital and Disaster Medicine. 2016;31(2):228-34.

64. Palma A, Ventura M, Galindo L, Fonseca F, Grifell M, Quintana P, et al. Something new about something old: a 10-year follow-up on classical and new psychoactive tryptamines and results of analysis. Journal of Psychoactive Drugs. 2017;49(4):297-305.

65. Palamar J, Acosta P, Sutherland R, Shedlin M, Barratt M. Adulterants and altruism: a qualitative investigation of "drug checkers" in North America. International Journal of Drug Policy. 2019;74:160-9. 66. Peiper N, Clarke S, Vincent L, Ciccarone D, Kral A, Zibbell J. Fentanyl test strips as an opioid overdose prevention strategy: findings from a syringe services program in the Southeastern United States. International Journal of Drug Policy. 2019;63:122-8.

67. Pérez S, de Dios M, Monteagudo E, Sanagustín D, Trabsa A, Grifell M, et al. New designer benzodiazepines use in Barcelona. European Psychiatry. 2017;41(S1):S874.

68. Quintana P, Ventura M, Grifell M, Palma A, Galindo L, Fornís I, et al. The hidden web and the fentanyl problem: detection of ocfentanil as an adulterant in heroin. International Journal of Drug Policy. 2017; 40:78-83.

69. Quintana P, Grifell M, Palma A, Fornís I, Gil C, Caudevilla F, et al. Is methylphenidate a prescription drug being sold in the illegal market? Analysis of samples submitted to a drug checking facility. European Psychiatry. 2016;33(S1):S313. 
70. Quintana P, Grifell M, Palma A, Gil C, Fornís I, Caudevilla F, et al. Presence of 2, 5-dimethoxy-4bromoamphetamine (DOB) among samples brought for drug checking in Spain. European Psychiatry. 2016;33(S1):S171.

71. Quintana P, Palma A, Grifell M, Tirado J, Gil C, Ventura M, et al. Is this really ketamine? The presence of methoxetamine in drug samples delivered as ketamine for substance analysis. European Neuropsychopharmacology. 2015;25:S621.

72. Roldán M, Grifell M, Gonzalez I, Fuentes J, Frings M, Ventura M, et al. Analysing heroin samples as harm reduction intervention - prevalence of adulteration with fentanyl derivatives. European Neuropsychopharmacology. 2019;29:S474-S5.

73. Sage C. Harm Reduction and Drug Checking; A wrap-around service for festivals. Case Study: Shambhala Music Festival /ANKORS Drug Checking Harm Reduction Service data 2015. ANKORS (AIDS Network Outreach \& Support Society); 2016.

74. Saleemi S, Pennybaker S, Wooldridge M, Johnson M. Who is 'Molly'? MDMA adulterants by product name and the impact of harm-reduction services at raves. Journal of Psychopharmacology. 2017;31(8):1056-60.

75. Sanagustín D, de Dios M, Monteagudo E, Pérez S, Trabsa A, Galindo L, et al. U-47700: the new emerging opioid drug. European Psychiatry. 2017;41(S1):S395.

76. Sande M, Šabić S. The importance of drug checking outside the context of nightlife in Slovenia. Harm Reduction Journal. 2018;15(2):1-8.

77. Sherman S, Morales K, Park J, McKenzie M, Marshall B, Green T. Acceptability of implementing community-based drug checking services for people who use drugs in three United States cities: Baltimore, Boston and Providence. International Journal of Drug Policy. 2019;68:46-53.

78. Sibbald K, Rushton W, King J, Charlton N. Adulterants in tablets sold as Ecstasy in the US from 20092013. Clinical Toxicology. 2014;52(7):704-5.

79. Spruit I. Monitoring synthetic drug markets, trends, and public health. Substance Use \& Misuse. 2001;36(1-2):23-47.

80. Spruit I. Ecstasy use and policy responses in the Netherlands. Journal of Drug Issues. 1999;29(3):65377.

81. Tanner-Smith E. Pharmacological content of tablets sold as "ecstasy": results from an online testing service. Drug and Alcohol Dependence. 2006;83(3):247-54.

82. Trabsa A, Monteagudo E, Mariona D, Grifell M, Galindo L, González L, et al. What are sweet dreams made of? Analysis of psychoactive substances into "gummies": a retrospective descriptive study. European Psychiatry. 2017;41(S1):S880.

83. Tupper K, McCrae K, Garber I, Lysyshyn M, Wood E. Initial results of a drug checking pilot program to detect fentanyl adulteration in a Canadian setting. Drug and Alcohol Dependence. 2018;190:242-5.

84. Valente H, Martins D, Carvalho H, Pires C, Carvalho M, Pinto M, et al. Evaluation of a drug checking service at a large scale electronic music festival in Portugal. International Journal of Drug Policy. 
2019;73:88-95.

85. van der Gouwe D, Brunt T, van Laar M, van der Pol P. Purity, adulteration and price of drugs bought online versus off-line in the Netherlands. Addiction. 2017;112:640-8.

86. van de Wijngaart G, Braam R, de Bruin D, Fris M, Maalsté N, Verbraeck H. Ecstasy use at large-scale dance events in the Netherlands. Journal of Drug Issues. 1999;29(3):679-701.

87. van de Wijngaart G, Braam R, de Bruin D, Fris M, Maalsté N, Verbraeck H. Ecstasy and the Dutch rave scene: A socio-epidemiological study on the nature and extent of, and the risks involved in using ecstasy and other party drugs at dance events. Addiction Research Centre. 1998.

88. Vidal C, Ventura M, Fornís I, Gil C, Calzada N, Fitó A, et al. Crystals and tablets in the Spanish ecstasy market 2000-2014: are they the same or different in terms of purity and adulteration? Forensic Science International. 2016;263:164-8.

89. Vidal C, Fornís I, Ventura M. New psychoactive substances as adulterants of controlled drugs. A worrying phenomenon? Drug Testing and Analysis. 2014;6(7-8):819-24.

90. Vogels N, Brunt T, Rigter S, van Dijk P, Vervaeke H, Niesink R. Content of ecstasy in the Netherlands: 1993-2008. Addiction. 2009;104:2057-66. 